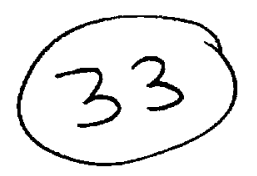

\title{
IMPLICATIONS OF THE OBSERVED MESOSCALE VARIATIONS OF CLOUDS FOR EARTH'S RADIATION BUDGET
}

\author{
William B. Rossow \\ NASA Goddard Institute for Space Studies \\ New York, NY \\ Carl Delo and Brian Cairns \\ Applied Physics and Mathematics \\ Columbia University at NASA GISS \\ New York, NY
}

May 2001 


\begin{abstract}
The effect of small-spatial-scale cloud variations on radiative transfer in cloudy atmospheres currently receives a lot of research attention, but the available studies are not very clear about which spatial scales are important and report a very large range of estimates of the magnitude of the effects. Also, there have been no systematic investigations of how to measure and represent these cloud variations. We exploit the cloud climatology produced by the International Satellite Cloud Climatology Project (ISCCP) to: (1) define and test different methods of representing cloud variation statistics, (2) investigate the range of spatial scales that should be included, (3) characterize cloud variations over a range of space and time scales covering mesoscale (30 - 300 $\mathrm{km}, 3-12 \mathrm{hr}$ ) into part of the lower part of the synoptic scale (300 - 3000 km, 1-30 days), (4) obtain a climatology of the optical thickness, emissivity and cloud top temperature variability of clouds that can be used in weather and climate GCMs, together with the parameterization proposed by Cairns et al. (1999), to account for the effects of small-scale cloud variations on radiative fluxes, and (5) evaluate the effect of observed cloud variations on Earth's radiation budget. These results lead to the formulation of a revised conceptual model of clouds for use in radiative transfer calculations in GCMs. The complete variability climatology can be obtained from the ISCCP Web site at http://isccp.giss.nasa.gov.
\end{abstract}




\section{Introduction}

Calculating radiative transfer in cloudy atmospheres is challenging because clouds introduce inhomogeneities of the optical medium, and hence, of the absorption, emission and scattering of radiation, over a very wide range of space and time scales. The long history of visual observations of clouds from the surface and aircraft has emphasized some basic characteristics in the representation of cloud variations in radiative transfer models (RTMs). The most basic division of cloud type names, cumulus and stratus, highlights the contrast between clouds that occur as "isolated" elements with similar horizontal and vertical dimensions and clouds that occur in extensive layers with horizontal dimensions that are very much larger than their vertical dimension. Cumulus also highlight the concept of highly variable and partial areal cloud cover, while stratus highlight the dramatic difference between clear and cloudy conditions. Because the development of RTMs, especially for use in global atmospheric circulation models (GCMs), has always been strongly constrained by computational limits, the best current, practical representation of clouds at larger scales emphasizes the "thin" layer structure by using planeparallel layers that are horizontally uniform at smaller (subgrid) spatial scales except for the variation of areal cover. The vertical, time and larger-scale horizontal variations are then treated explicitly by varying the properties and areal coverage of these cloud layers. The advent of the doubling-adding technique (Hansen and Travis 1974) allowed for very detailed, but still practical, treatment of the vertical variations of atmospheric properties (e.g., Lacis and Oinas 1997), but little advance over the plane-parallel representation of clouds has been made until recently.

Efforts continue to understand the consequences of smaller-scale horizontal inhomogeneity for radiative fluxes, particularly inspired by the occurrence of the "non-layer" cloud types (called the 3-D problem to contrast it with the 1-D plane-parallel treatment), and to re-examine the relationships among cloud water content, radiation and atmospheric dynamics at the smallest scales, stimulated by the advent of prognostic cloud water schemes in GCMs (e.g., Sundqvist 1978, Smith 1990, Tiedtke 1993, Del Genio et al. 1996, Fowler et al. 1996). The 
Page 2

remaining problem, once the water mass has been determined for each model layer in a GCM, is how to distribute the water, both horizontally in a given layer and vertically in terms of cloud layer overlaps (Li et al. 1994, Oreopoulos and Barker 1999). The simplest aspect of this dilemma how to average over a non-linear relationship - has sometimes been combined with the problem of treating the true 3-D cloud effects on radiative transfer (cf. Cahalan et al. 1994a, Barker 1996a), but this is not necessary. A brief summary of the many investigations of radiative transfer at smaller scales, mostly at solar wavelengths, is as follows. First, there is a smallest scale of cloud variability, 100-300 m, that is expressed in the shortwave radiation field (Cahalan 1989, Marshak et al. 1995, 1998b, Byrne et al. 1996, Davis et al. 1997). Second, unresolved variability in cloud properties can lead to a difference between the mean albedo of a scene and the albedo of the mean properties of the scene, which effect has been termed the plane parallel bias. Estimates of the magnitude of this effect vary over an albedo range from 0.025 to 0.30 (Davies 1978, Hignett 1987, Barker and Davies 1992, Kobayashi 1993, Cahalan et al. 1994a, 1995, Coley and Jonas 1996, Loeb and Coakley 1998, Marshak et al. 1998a, Oreopoulos and Davies 1998a, Barker et al. 1999), which has been discussed by Pincus et al. (1999). Third, there are a number of different methods that have been proposed for representing small-scale cloud variability and parameterizing its effect on radiative fluxes (Stephens 1988a,b, Kobayashi 1991, Cahalan et al. 1994b, Barker 1996a,b, Barker et al. 1996, Oreopoulos and Davies 1998b, Barker and Fu 1999, Cairns et al. 2000).

Satellite observations have provided a somewhat different perspective on some of these issues. First, satellite observations show that cloud properties actually vary over a very much larger range of spatial scales than can be observed from the surface or aircraft, essentially covering all spatial scales up to planetary scales (Rossow and Cairns 1995). Moreover, the power spectrum of these cloud variations is continuous with a negative slope: i.e., the amplitude of cloud variations is much larger at the larger scales of the atmospheric general circulation than it is at the smaller ("turbulent") scales usually observed by surface or aircraft observers. Second, the satellite 
viewpoint suggests that the scattered and broken cloud types actually tend to occur over areas that are much larger in scale than the individual cloud elements (Seze and Rossow 1991b) with a variety of organizations at intermediate scales (e.g., Ramirez and Bras 1990, Nair et al. 1998). Consequently, these cloud types could actually be considered to be layers that are "broken" by small-scale regions of zero cloud mass density (i.e., clear sky). This interpretation is reinforced by the observation that, when cloud cover falls below $100 \%$ on scales of order $100 \mathrm{~km}$, the average cloud mass density is also much lower (Barker et al. 1996, Considine et al. 1997). As Cahalan et al. (1994a) pointed out, the effects of horizontal variability are not only still present for complete overcast, they are larger in magnitude because the average mass density and its range of variation is larger. Thus, we could say that all clouds occur in physically thin $(<3000 \mathrm{~m}$, Wang et al. 2000) layers (the notable exception being penetrating convective towers) and that there is only one radiative transfer problem of accounting for the effects of smaller-scale horizontal cloud variability.

The variety of approaches used and the wide range of results obtained in these studies have left some questions unanswered. What is the most effective way to represent small-scale cloud variations in radiative flux calculations? How do we measure cloud variations on the requisite scales? Precisely what range of spatial scales of cloud variability should be included? Does a useful distinction between cloud cover and cloud mass density variations exist at smaller scales (cf. Rossow 1989, Byrne et al. 1996)?

In this paper, we use the International Satellite Cloud Climatology Project (ISCCP) datasets to address these questions. In Section 2 we define a general variability statistic for optical media at the smallest scales, discuss the range of spatial scales that should be included in determining its magnitude, define a satellite-based measure of cloud variability and calculate different measures of the effects of small-scale cloud variability on radiative fluxes. This discussion leads to the formulation of a revised conceptual model of clouds at the end of Section 2. In Section 3 we use the most detailed ISCCP dataset to examine the details of cloud variations 
over the range of space and time scales covering the mesoscale (30 - 300 km, 3-12 hr) into the lower part of the synoptic scale range (300-3000 km, 1-30 days) and to test our satellite-based measure of variability effects on radiative fluxes (this study is supplemented by an Appendix). In Section 4 we present a climatology of the mesoscale variability of cloud optical thickness, emissivity and temperature that can be used in current GCMs to test and refine their treatment of cloud-radiation interactions. In Section 5 we quantify the effects of the small-scale cloud variability on Earth's radiation budget and Section 6 discusses some additional implications for modeling. 


\section{Representing Cloud Variability}

a. Definition of the variability statistic

Since our purpose is to provide a climatological quantification of mesoscale (i.e., global circulation model subgrid-scale) cloud variability, we seek statistical representations of the radiative impacts of inhomogeneous clouds: quantitative measures of the albedo and emissivity biases that would occur if naturally occurring clouds were treated as horizontally homogeneous at these smaller scales. This statistical approach is also a more practical way to account for the systematic effects of smaller-scale cloud variations; rather than insisting on high accuracy in the representation of the particular details of particular clouds, we parameterize the radiative effects of a statistical aggregate. This approach differs from some previous studies (e.g., Barker 1996a, Barker et al. 1999), which have tried to derive analytical models of the optical thickness distributions for specific cloud regimes (e.g., marine stratus, fair-weather cumulus, etc). The best measure of cloud variability should be generally applicable without regard to location, season and cloud type. In particular, we avoid methods that assume a specific analytical shape for the optical thickness distributions (e.g., log-normal, exponential, gamma, see the Appendix).

The measure of variability is based on Cairns et al. (2000), who propose a correction factor, $\epsilon_{p}$, derived for a generalized inhomogeneous cloud particle density distribution, that is used to modify the radiative transfer equations for clouds being treated as plane-parallel for computational convenience (the subscript, $\mathrm{p}$, emphasizes that this quantity relates to the cloud particle density distribution). The factor, $\epsilon_{\mathrm{p}}$, is derived for a randomly varying, isotropic, threedimensional density distribution and is used to re-normalize the radiative parameters of the cloud: extinction cross-section $\left(\sigma_{\text {ext }}\right)$, single-scatter albedo $\left(\varpi_{0}\right)$ and asymmetry parameter $(\mathrm{g})$. The radiation field of an inhomogeneous cloud is then calculated for an equivalent homogeneous cloud with these re-scaled parameters. The re-normalization for extinction cross-section for a randomly varying cloud medium with relatively weak variability is (see Cairns et al. 2000 for the scaling for $\varpi_{0}$ and $\left.\mathrm{g}\right)$ : 


$$
\sigma_{e x t}^{\prime}=\left(1-\varepsilon_{p}\right) \sigma_{e x t}
$$

with

$$
\varepsilon_{p}=\frac{1}{2}(\alpha-\sqrt{\alpha-4 V})
$$

and

$$
\alpha=\frac{1+\sigma_{e x t} l_{c}}{\sigma_{e x t} l_{c}}
$$

where $\mathrm{V}$ is the relative variance of the particle density distribution (variance divided by the square of the mean density) and $l_{\mathrm{c}}$ is the effective correlation length of the variations. This parameterization has the obvious consequence that variability on scales smaller than the transport mean free path has no effect on the mean radiative properties of the cloud (cf. Marshak et al. $1995,1998 \mathrm{~b})$. In cases where the correlation length is of the same order as the photon mean free path $\left(\sigma_{\mathrm{ext}} l_{\mathrm{c}} \approx 1\right)$ and the particle density fluctuations have log-normal statistics, the renormalization depends solely on the statistics of the cloud particle density distribution.

b. Extension from small to larger scales

To evaluate the effects of cloud variability on radiative fluxes, (1) can be transformed to define an effective optical thickness, $\tau$ ', for a varying particle density distribution:

$$
\tau^{\prime}=\left(1-\varepsilon_{p}\right) \tau
$$


where

$$
\tau \equiv \int \sigma_{e x t}(\zeta \mathbf{z}) d \zeta=\mathrm{Y}_{\mathbf{z}}=\cos \theta \int \sigma_{e x t}(\zeta \mathbf{s}) d \zeta=\cos \theta \mathrm{Y}_{\mathbf{s}}
$$

Here $\mathrm{Y}$ is the optical path (the line integral of extinction) and $\mathbf{z}$ and $\mathbf{s}$ are unit vectors in the vertical and in any arbitrary direction. This definition of total optical thickness follows Goody and Yung (1989) and the last part of (4) is formally valid only for a stratified atmosphere in which the extinction varies only in the vertical direction (Figure 1a). In this idealized situation there is no restriction on the horizontal length scale over which optical thickness is well-defined since the line integrals of extinction over the vertical and over all slant paths are simply related as in (4). Thus, in this case the optical thickness is unambiguous and can be determined from the optical path evaluated at an arbitrary angle $\left(\mathrm{Y}_{\mathrm{z}}\right.$ or $\left.\mathrm{Y}_{\mathrm{s}}\right)$. However, horizontal variability in the extinction field destroys this relation (Figure $1 b$ ), where now the extinction, $\sigma_{\mathrm{ext}}(\mathbf{x}, \mathbf{z}, \lambda)$, varies horizontally with a characteristic length scale, $\lambda$, and a space-borne instrument performs an integration along the line of sight and over an area defined by the instrument's field-of-view, represented by a length, $\mathrm{L}$. Now the optical thickness cannot be simply related to the spatially varying optical path values as in the ideal case. In such a situation it is no longer clear what the proper definition of optical thickness should be. Reasonable choices might be the vertically or hemispherically integrated extinction averaged over $\lambda$ or $L$.

However, it is much simpler to restore the relation between optical thickness and optical path embodied in (4) by replacing the condition of absolute horizontal homogeneity with one of statistical homogeneity. This situation is shown in Figure 1c, where the remotely-sensed optical path is evaluated over a region that is large compared to the scale of the horizontal variability, i.e., $\mathrm{L} » \lambda$, 


$$
\tau_{L}=\frac{\int \sigma_{e x t}(L \mathbf{x}, \zeta \mathbf{z}, \lambda) d \zeta d L d L}{\int d L d L} \approx \frac{\cos \theta \int \sigma_{e x t}(L \mathbf{x}, \zeta \mathbf{s}, \lambda) d \zeta d L d L}{\int d L d L}
$$

In essence the process is simplified by letting the satellite instrument do the integration (averaging) over an appropriate scale. All that remains is to specify a reasonable lower bound for $\lambda$, along with a condition on $L$ (e.g., $L \geq 10 \lambda$ ).

An important conclusion from this discussion is that there is a minimum horizontal scale at which the optical thickness is a meaningful quantity. This scale is imposed by the interaction of the radiation field with the inhomogeneous extinction field: when 3-D radiative effects are important, then optical thickness is not a meaningful parameter. An additional implication is that any quantities derived from the optical thickness will be subject to the same resolution requirements.

Assuming we are working at an appropriate scale, $L$, then $\tau$ ' can be interpreted as the mean optical thickness of a spatially variable medium computed on a radiative basis, e.g., as an albedo-preserving radiative mean value. Therefore, $\epsilon_{\mathrm{p}}$ is a direct measure of the extent to which the variability of the particle density distribution reduces the effective optical thickness, and hence the spherical albedo, of a cloudy scene relative to the homogeneous situation. Note the formulation of Cairns et al. (2000) makes no assumptions about the shape of the cloud particle density distribution, so that, although the relationship between $\epsilon_{\mathrm{p}}$ and the particle density distribution depends on the shape of distribution, using $\epsilon_{\mathrm{p}}$ directly to correct the radiative transfer for inhomogeneities in the medium is completely general (cf. Oreopoulos and Davies 1998b).

c. Physical domain of validity

Since the magnitude of cloud variations is scale-dependent, we must decide what range of 
spatial scales to include in determining the radiative fluxes. The negative slope of the power spectrum of cloud spatial variations means that the choice of the upper scale limit determines the magnitude of any variability statistic; however, we also need sufficient sampling of the smaller scales for an accurate statistical estimate. We also need to make measurements of the radiances at scales large enough that the 3-D effects do not prevent a meaningful measure of cloud optical thickness. Thus there are two separate issues: the best scales for retrieval of cloud optical thickness and its variations from satellite radiance measurements and the best scales at which to calculate radiative fluxes with parameterized smaller-scale variability.

\section{i. Lower scale limits}

The smallest scale that needs to be considered is set either by the smallest scale at which cloud properties vary significantly or the smallest scale at which the photons can respond to cloud variations, whichever is larger. Aircraft observations of cloud variations along flight paths, although not systematically analyzed, suggest that significant cloud property variations appear only on scales greater than about 3 - 30 m (e.g., Lilly and Petersen 1983, Korolev and Mazin 1993, Davis et al. 1999 and references therein). This is consistent with the scales of the turbulent motions in the planetary boundary layer that exhibit dominant eddy sizes of 1-10 km and power spectrum slopes of $-5 / 3$ (cf. Lilly 1983). The implication is that the motions at scales $<100 \mathrm{~m}$ are at least two orders of magnitude smaller than those at the dominant scale, i.e., substantially less than $1 \mathrm{~cm} / \mathrm{s}$. Such very weak motions cannot produce substantial gradients in atmospheric temperature and humidity sufficient to produce variations in cloud particle properties (Jonas 1994).

Using the transparent approximation (e.g., Goody and Yung 1989, pp. 53-54), it can be shown that the spatial variations of the optical properties of clouds smaller than the photon mean free path are averaged out by the radiation field (Marshak et al. 1998b). Detailed Monte Carlo calculations show that the mean free path for solar wavelength photons in Earth's cloudy 
atmosphere is about $100 \mathrm{~m}$ and that the effective "coupling" scale where the radiation field expresses variations in the optical medium is about $300 \mathrm{~m}$ (Marshak et al. 1995, Davis et al. 1997). Although the coupling scale for more strongly absorbed infrared photons is somewhat smaller, variations of atmospheric temperature are governed by the turbulent motions that occur at scales larger than about $100 \mathrm{~m}$ (cf. Lilly 1983). As stated above the formulation of (1) embodies this radiative smoothing. Thus, even if substantial cloud mass variations were to occur at scales $<100-300 \mathrm{~m}$, the radiation field would not exhibit such scales because the pathlength required for the photons to interact is larger. In other words, the cloud is effectively homogeneous at the smallest scales (cf. Marshak et al. 1995, Byrne et al. 1996).

Another crucial implication of this result is that one cannot determine where the edge of a cloud is to a precision better than about 100-300 m using radiation observations (cf. Rossow 1989); thus concern about "sub-pixel" cloud cover at scales smaller than this is misplaced.

\section{ii. The sampling scale, $\mathrm{L}$}

Much recent research (e.g., Barker and Liu 1995, Barker et al. 1996, Chambers et al. 1997a) has involved estimating the magnitude of optical thickness variability by analysis of very high spatial resolution satellite radiance data (e.g., 30 m LANDSAT data), where the retrieval procedure assumes each $30 \mathrm{~m}$ pixel is "plane-parallel" and independent of its neighbors, often called the "independent pixel approximation" (IPA). From a consideration of Figure 1, it is evident that the condition for IPA to be satisfied exactly is absolute homogeneity (Figure 1a), which might be true at $30 \mathrm{~m}$ scale, but that the lateral transport of photons at this scale, which is smaller than the mean free path, is dominant. However, even though IPA has been shown to provide a good estimate of the spatially-averaged fluxes through a variable medium, the detailed calculations of Marshak et al. $(1995,1998 \mathrm{~b})$ show that the neighboring pixels at scales $\leqslant 300 \mathrm{~m}$ cannot be considered independent in a radiative sense and that use of optical thickness distributions derived from radiance analyses with IPA at such small scales actually overestimates 
the radiative variability compared with Monte Carlo calculations. The other possibility illustrated in Figure $1 \mathrm{c}$ is that IPA can be a satisfactory approximation for a medium that is statistically homogeneous, but only at the larger scale, L. In other words, the conditions necessary for the validity of IPA are equivalent to those needed for an unambiguous determination of optical thickness. Marshak et al. (1995) show that the optical thickness retrievals are better when done at a scale of several kilometers (about ten times the coupling scale) where the 3-D effects are reduced and the lateral photon exchanges begin to cancel out. Barker (1995) shows empirically that a better correspondence between cloud albedo and optical thickness appears only at larger scales. Orecpoulos and Davies (1998b) also suggest that the small-scale effects are reduced at scales of several kilometers because sub-pixel cloud cover reduces the mean optical thickness; but, as discussed above, it is not obvious that cloud cover has much meaning at scales smaller than the coupling scale. Their results suggest instead that the retrieved optical thicknesses represent the spatial distribution better if the radiation is averaged over a few kilometers scale and they show that the magnitude of the remaining 3-D effects at $5 \mathrm{~km}$ is no more than about $1 \%$. Observed power spectra of radiances already demonstrate that the magnitude of the contributions from scales smaller than a few kilometers are negligible (Chambers et al. 1997b), consistent with an extrapolation of the cloud variations in the ISCCP data from the observed scales to sub-pixel scales (here $<5 \mathrm{~km}$ ).

As we have noted above, optical thickness cannot be meaningfully defined from radiance measurements at scales where the 3-D effects dominate. Moreover, any study that uses data from LANDSAT or any instruments on polar orbiting satellites, also suffers from the problem that the coupling of space and time scales cannot be observed since all one has is a snapshot of the spatial structure. Use of such snapshots may distort our understanding of the effects of small-scale cloud variability (we discuss this point more in Section 6).

iii. Cloud optical thickness variability and cloud cover 
The physical limit on optical thickness calculations should propagate through the analysis consistently, i.e., those derived quantities that depend on optical thickness are also subject to the same spatial scale limitations (cf. Di Girolamo and Davies 1997). In particular, the concept of "sub-pixel cloud fraction," invoked in some studies (e.g., Loeb and Davies 1997, Loeb and Coakley 1998), is probably moot. Very small-scale cloud fraction effects certainly exist at the scales where the radiative field is 3-D (Korolev and Mazin 1993, Kobayashi et al. 1999, 2000); however, if the radiation is essentially averaging over the smaller scales, the result may simply be that the radiation does not "see small holes" in the droplet density distribution, even if they are present (cf. Byrne et al. 1996, Marshak et al. 1998b). In other words, variations of optical thickness, including zero values, may not be important at scales $\leqslant 300 \mathrm{~m}$. Barker and Wielicki (1997) show this also true at thermal infrared wavelengths. Detailed analysis of high resolution ( $30 \mathrm{~m}$ ) observations of the quintessential case, marine boundary layer clouds, by Wielicki and Parker (1992) and Chambers et al. (1997b) shows that, in fact, most of the radiance variations observed at few-kilometer scales is caused by variations of cloud optical thickness, not cloud cover, and that this becomes even more pronounced when the cloud cover falls below $100 \%$. Thus, the cloud fraction defined by radiative measurements should, like the optical thickness, only be determined at scales at least ten times larger than the photon coupling scale.

\section{iv. Upper scale limits}

One way to set the upper scale limit is to determine the size of the area that contributes significantly to the flux at a particular point. Figure 2 illustrates the geometry for determining the contribution of each element of area as a function of the height above the radiating surface. We can easily account for the change of projected area with viewing zenith angle, $\theta$, but neglect the more complicated treatment for the variation of radiation over a range of altitudes above the surface since the magnitude of the radiation generally decreases with height (especially for longwave fluxes). We also neglect attenuation of radiation coming from the surface, which 
increases as the pathlength, $d$, increases towards the edge of the viewed region. All of these simplifications mean that our estimate of the area contributing to the flux is a slight underestimate. The quantitative answer then depends on what height we choose and what fraction of the total flux we consider sufficient. About $99 \%$ of the total atmospheric mass and, therefore most of the radiation, lies below a height of about $30 \mathrm{~km}$. Radiative heating at higher levels would have contributions from a larger area, whereas radiative heating at lower levels and at the surface looking upward involve smaller contributing areas. For an upwelling flux at a height of $30 \mathrm{~km}$, about two-thirds of the total comes from an area with a radius of about $200 \mathrm{~km}$. Thus, the radiative fluxes at a particular location can be considered independent of the neighboring conditions at scales of about $300-500 \mathrm{~km}$.

Another argument for selecting the "best" scale for treating the radiation using the scheme proposed here to account for smaller-scale variations comes from the fact that the representation of the cloud variability is inherently statistical. Consequently, the parameter used to represent the cloud variability will not be accurate when applied to too small a sample population. Given that the optical thickness and cloud cover are best defined from measurements at scales $21-3 \mathrm{~km}$ and that the radiation efficiently couples to the atmospheric motions only at scales larger than the smaller turbulent motions (i.e., $1-10 \mathrm{~km}$ ), an appropriate sample of variations at these scales would be obtained for domain sizes of at least 100-300 km in size. Marshak et al (1998b) and Barker et al. (1999) reach a similar conclusion by showing that specific 3-D geometric effects appear in the radiance fields at scales $<10 \mathrm{~km}$ but that average fluxes are well-represented at scales of $100 \mathrm{~km}$ using a statistical representation of the optical thickness variations. Lenschow and Stankov (1986) have directly evaluated the scales necessary to estimate cloud variability accurately from long aircraft transects (see Astin and Di Girolamo 1999 for a theoretical discussion). They find that variances are determined within $10 \%$ only when measurements are collected over transect lengths 10 - 100 times the boundary layer depth (roughly the dominant horizontal eddy size as well). This implies that the statistics of cloud variations are meaningfully 
represented only at scales $\sim 10-100 \mathrm{~km}$. For convenience, then, we will use the ISCCP grid resolution, about $280 \mathrm{~km}$, as the upper scale limit.

d. Formulation of $\epsilon$ for satellite data

The generality of the correction in (3) led us to formulate a version of $\epsilon_{\mathrm{p}}$ appropriate for larger-scale, remotely sensed cloud fields, where the integration of extinction cross section into optical thickness is statistically valid. Interpretation of the correction parameter, $\epsilon$, in terms of correlation length scales and the moments of the cloud particle density distribution is no longer completely appropriate as a large-scale, derived statistic since it would depend explicitly on the variability that exists between the scale of individual pixels and the size of the domain over which the statistics are accumulated. In this respect, $\epsilon$ is only an analog to the microphysical parameter, $\epsilon_{\mathrm{p}}$; however, $\epsilon$ is the statistical generalization of $\epsilon_{\mathrm{p}}$ that can actually be determined from observations (cf. Cahalan 1994b, Oreopoulos and Davies 1998b). The validity of $\epsilon$ will depend directly on the accuracy of retrieving optical thicknesses from the pixel-level radiance measurements using IPA, i.e, assuming that the net lateral transport of photons between pixels is zero, and on obtaining a sufficient sample of the cloud variability present. From Figure $1 \mathrm{c}$ and the discussion that followed, we consider radiances averaged over the scale, $\mathrm{L} \approx 5 \mathrm{~km}$, the same scale at which the determination of optical thickness is unambiguous, and collect a sample population over a scale of $280 \mathrm{~km}$.

For clarity in the following discussion, we define three different first moments of a discrete, normalized optical thickness distribution, $p\left(\tau_{j}\right)$ :

Linear mean:

$$
\bar{\tau}=\sum p\left(\tau_{i}\right) \tau_{i}
$$




$$
\hat{\tau}=R^{-1}\left\{\sum p\left(\tau_{i}\right) R\left(\tau_{i}\right)\right\}=R^{-1}\left\{\overline{R\left(\tau_{i}\right)}\right\}
$$

Logarithmic mean:

$$
\widetilde{\tau}=\exp \left\{\sum p\left(\tau_{i}\right) \ln \tau_{i}\right\}=\exp \left\{\overline{\ln \tau_{i}}\right\}
$$

where $\mathrm{R}$ is the radiative transfer operator that determines a radiative flux from the optical thickness. These averages are calculated for all non-zero values of optical thickness (cloudy pixels only). The linear mean optical thickness, $\bar{\tau}$, is proportional to cloud water path (ISCCP reports this value as water path), which may be predicted in a model cloud physics subroutine. This value is also often referred to as the "plane-parallel" or "homogeneous" optical thickness. The radiative mean, $\hat{\tau}$, reported by ISCCP as the mean optical thickness, is calculated with weights that are linear in spherical albedo, so that it represents the (lower) optical thickness of a homogeneous cloud that has the same albedo as the actual inhomogeneous cloud with $\bar{\tau}$. The logarithmic mean, $\tilde{\tau}$, is roughly equivalent to the radiative mean and is used in an Appendix to examine gamma-distribution-based parameterizations of the effects of cloud inhomogeneity. The $\tau_{i}$ values are obtained for individual satellite pixels (in the ISCCP DX dataset, these represent areas about 5 $\mathrm{km}$ in size, sampled at intervals of about $30 \mathrm{~km}$ ). In the ISCCP gridded datasets, the linear and radiative mean values are computed and reported for all cloudy pixels in an area of about (280 $\mathrm{km})^{2}$ every $3 \mathrm{hr}$ (D1 data) or every month (D2 data). For some of the results shown later, the three mean values are computed directly from the DX data over a range of space and time scales with variable map grid size.

The radiative mean optical thickness in (7) is taken to correspond to $\tau$ ' defined in (3) since 
it is, by definition, the optical thickness of the homogeneous cloud that has the same albedo as the inhomogeneous cloud field. For satellite data, if we assume that the linear and radiative means can be obtained from (6) and (7), then we can define a variability statistic of the inhomogeneous cloud field in analogy with (3) as

$$
\varepsilon=1-\frac{\hat{\tau}}{\bar{\tau}}
$$

Thus, $\epsilon$ serves directly as the descriptor of the radiative variability of an arbitrary, remotelysensed optical thickness distribution. For a homogeneous distribution, $\epsilon=0$; as the variability and its radiative effect increase, then $\epsilon$ increases towards 1 . Note that $\epsilon$ does not depend on the shape of the optical thickness distribution since it can be determined directly from the observed $\tau$ values. As discussed above and shown in the next section, $\epsilon$ varies with the range of space and time scales included to produce the distribution. The correction to the mean optical thickness, needed to account for cloud inhomogeneity, is then given by (cf. Cairns et al. 2000)

$$
\hat{\tau}=(1-\varepsilon) \bar{\tau}
$$

Our statistic, $\epsilon$, is, in fact, defined in the same manner as the reduction factor, $\chi_{0}$, in Eq. (7) of Oreopoulos and Davies (1998b), such that $\epsilon=1-\chi_{0}$. Their reduction factor is an extension of the Effective Thickness Approximation (ETA) of Cahalan et al (1994b), viz., $\overline{\log \tau_{i}}=\log [\chi(\bar{\tau})]$, which in the notation used here is equivalent to $\chi=\tilde{\tau} / \bar{\tau}$. In portions of the optical thickness-albedo relation, where the radiative weighting is nearly logarithmic (the 
lower range of optical thicknesses encompassing most of the clouds), $\chi_{0}$ and $\chi$ will be very similar. Larger deviations between these two quantities are found for distributions with very high variances, i.e., very "wide" distributions usually involving a few very thick (e.g., convective) clouds mixed with very thin clouds (i.e., cirrus). For such distributions $\tilde{\tau}$ and $\hat{\tau}$ will diverge.

It is encouraging to see that the parameter, $\epsilon$, has arisen independently, from the present extension of a self-consistent, generic correction to radiative transfer through inhomogeneous media and from a realization that a proper quantification of variability should be derived from its actual impact on the physical world in Oreopoulos and Davies (1998b). In the latter work, the variation of $\chi$ and $\chi_{0}$ with region size was only briefly discussed. Here we take $\epsilon$ as the fundamental variability statistic and examine its behavior as a function of space and time scale and produce a climatology of its value from the ISCCP dataset.

To make connection with other parameterizations of cloud variability effects on radiative fluxes (e.g., Barker 1992, 1996a, Cahalan et al. 1994b, Barker and Wielicki 1997, Oreopoulos and Davies 1998a, b, Barker et al. 1999), we have also tested methods for determining gamma function fits to the observed distributions of cloud optical thickness (Appendix).

e. Evaluation of homogeneous biases and extension to infrared wavelengths

The primary modeling problem is to relate cloud properties, observed or predicted by the cloud physics subroutine in a GCM, to the radiative fluxes. The most direct method is to use the cloud water content, expressed in terms of particle number density, size, shape and phase, together with its vertical variation and the physical thickness of the cloud layer, to determine the optical thickness, single scattering albedo and asymmetry parameter of the cloud. The variability correction factor, $\epsilon$, can then be used in the scheme proposed by Cairns $e t$ al. (2000) to re-scale these optical parameters (see Section 5). Another approach is to use this information to determine the reflectivity (albedo), transmissivity, and absorptivity/emissivity of the cloud by some direct 
relationship with water content, so we also present the inhomogeneity correction in terms of albedo and emissivity.

For a cloudy scene, the plane-parallel, homogeneous albedo bias can be calculated from

$$
A_{p p}-A=R\{\bar{\tau}\}-R\{\hat{\tau}\}=R\{\bar{\tau}\}-R\{(1-\varepsilon) \bar{\tau}\}
$$

where $A_{p p}$ is the plane-parallel cloud albedo, $A$ is the true albedo for an inhomogeneous cloud and $\mathrm{R}$ is the function relating optical thickness to albedo. Thus, the correction to the homogeneous albedo is given by

$$
A=R\{(1-\varepsilon) \bar{\tau}\}
$$

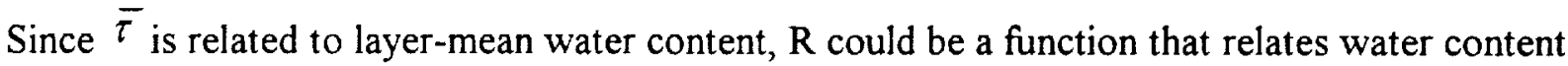
directly to cloud albedo (e.g., Stephens 1978). It is apparent from (11) that the bias can be wellrepresented by the specification of $\bar{\tau}$ and $\epsilon$. We show in the Appendix that this is a more stable and appropriate characterization of the effects of small-scale cloud variability on the radiation than other commonly proposed measures that use a second moment of the optical thickness distribution, e.g., $\sigma_{\tau}, \sigma_{\ln (\tau)},\left(\sigma_{\tau} / \bar{\tau}\right)$, etc. Our statistic is derived from two different first moments of the distribution and is therefore more robust in situations with limited sample population. Moreover for the highly skewed and/or multi-modal distribution shapes that are common for cloud optical thicknesses, it is not clear how quickly the second moment converges and how representative it is in such situations (Lenschow and Stankov 1986 and Seze and 
Rossow 1991 a both show from different datasets that the first moment of the distributions converges much more rapidly than the second moment). Using higher-order moments of the distribution is particularly problematic for scenes with mixtures of optically thin and optically thick clouds (i.e., cirrus and convection) that are common in the tropics. Since the radiative effect of changing distributions of optical thickness directly underlies the definition of $\epsilon$, it suffers less error in situations where a few optically thick clouds may dominate the second mornent or when low mean optical thickness may cause high values of the relative dispersion, $\left(\sigma_{\tau} / \bar{\tau}\right)$, as we show in the next section. In either case, a large second-moment estimate of the variability may not actually accompany a large absolute bias in albedo of the homogeneous estimate. Most importantly, no assumptions have been made in the formulation of $\epsilon$ about the shape of the optical thickness distributions and, since our variability statistic, $\epsilon$, can be determined directly from the observed distribution of satellite-measured radiances without assuming a specific distribution shape, the use of an analytic approximation to the distribution as an intermediate step in estimating albedo biases is not needed.

The parameterization of radiative variability effects can be extended to the longwave (IR) regime (cf. Barker and Wielicki 1997) by defining an analogous statistic:

$$
\varepsilon_{I R}=1-\frac{\hat{\tau}_{I R}}{\bar{\tau}_{I R}}
$$

where the effective infrared optical thickness, $\hat{\tau}_{I R}$, is determined from the observed distribution of optical thicknesses, $p\left(\tau_{i}\right)$, as before and therefore is valid for the same range of space and time scales. The emissivity, E, of the discrete distribution is then represented by 


$$
E=1-\sum\left[p\left(\tau_{i}\right) \exp \left(-b \tau_{i}\right)\right]=1-\overline{\exp \left(-b \tau_{i}\right)}=1-\exp \left(-\hat{\tau}_{I R}\right)
$$

where $b$ is a coefficient that relates visible optical thickness to infrared optical thickness as a function of cloud particle phase and the radiatively weighted optical thickness, $\hat{\tau}_{I R}$, is defined by the last equality. The plane-parallel, homogeneous emissivity, $\mathrm{E}_{\mathrm{pp}}$, is given by replacing $\hat{\tau}_{I R}$ by $\bar{\tau}_{I R}$ in the last part of (14). Thus, the emissivity bias is given by

$$
E_{p p}-E=-\exp \left(-\bar{\tau}_{I R}\right)+\exp \left[-\left(1-\varepsilon_{I R}\right) \bar{\tau}_{I R}\right]
$$

Although $\epsilon$ is a direct measure of the homogeneous albedo bias, large values of $\epsilon$ are not always indicative of large values of $\epsilon_{\mathrm{RR}}$ and a large emissivity bias. For optically thick clouds, the emissivity is essentially unity even if the optical thickness is varying a lot. This means that the effects of spatial variations of cloud optical thickness on the shortwave and longwave radiative fluxes will differ with cloud type.

In Section $3 \mathrm{~b}$, we show that a simple empirical relationship between $\epsilon$ and $\epsilon_{\mathrm{IR}}$ can be derived from the pixel-level cloud data. This analysis defines an approximate function:

$$
\begin{gathered}
\varepsilon_{I R}^{\prime}=1-x+\sqrt{\rho^{2}-(x-\varepsilon)^{2}} \\
x=\frac{1}{2}\left(1+\sqrt{2 \rho^{2}-1}\right)
\end{gathered}
$$


and $\rho=1+3.65 / \tau$ is the radius of a circle that passes through the points $(0,0)$ and $(1,1)$ and that fits the curvature of the relationship (see Figure 9). We discuss this relationship further in Section $3 b$.

\section{f. Summary}

The refined conceptual model we are proposing augments the "large-scale" cloud parameters needed for describing the radiative fluxes (cloud cover, optical thickness and temperature) by adding a measure of cloud optical thickness variability at "smaller scales" as another optical parameter (cf. Stephens 1988a, b, Tiedtke 1996). Small-scale cloud top temperature variability should also be added (cf. Stubenrauch et al. 1999). We consider these parameters to be "large-scale" in the sense that they are varied explicitly to represent the synoptic variations of the radiative properties of the atmosphere (i.e., cloud types) at all other scales larger than that of the model map grid. Cloud cover fraction has been used as a linear mixing parameter in radiative models to represent smaller-scale variability as a linear mix of the radiation for clear and overcast conditions (cf. Byrne et al. 1996). Our refinement amounts to expanding the representation beyond cloud cover to include variability within the cloud. This is the first-order approximation for small-scale variation effects (cf. Zuev and Titov 1995, Barker et al. 1999 and Kobayashi et al. 2000); but there are still weaker effects that depend explicitly on the size distribution of the cloud elements that is not accounted for by our parameterization. 


\section{Mesoscale Cloud Variability}

The discussion in the previous section suggests making measurements of cloud optical thickness at scales of a few kilometers, where statistical homogeneity can be assumed, and sampling the variability over scales of several hundred kilometers for radiative flux calculations. The ISCCP dataset reports optical thickness values for individual satellite pixels, about $5 \mathrm{~km}$ in size (averaged over all satellites) that have been sampled to spatial intervals of about $30 \mathrm{~km}$. Although this sample is a little sparse, Seze and Rossow (1991b) showed that the variability statistics are equivalent to the original dataset if collected over time periods of at least 10 days for a region size of $280 \mathrm{~km}$. Thus, the ISCCP dataset can be used to obtain a climatology of cloud variability at scales $>5 \mathrm{~km}$, which accounts for most of the variability (cf. Chambers et al. 1997b). The ISCCP gridded (D1) dataset includes values of both the linear and radiative mean optical thicknesses, from which we can calculate $\epsilon$ and $\epsilon_{\mathrm{IR}}$, as well as spatial standard deviations (Rossow et al. 1996). To show that the summary statistics for $280 \mathrm{~km}$ regions can accurately represent the radiative effects of cloud spatial variability, we examine sixteen different climate regimes in detail (Figure 3) using the ISCCP DX data for July 1992. We use the actual distributions of optical thickness and emissivity to determine the variation statistics and to calculate the average fluxes directly (assuming IPA) for comparison with the grid-box-average calculations. In the Appendix, we compare alternative methods for representing the variability in more detail.

\section{a. Cloud variation statistics}

Figure 4 summarizes the pixel-level distributions of cloud optical thicknesses in the sixteen areas, aggregated over a month. Some distributions, e.g., for marine boundary layer clouds (Figure 4: regions 2072, 2446, 2690, 4647 and 4940), are relatively narrow, exhibiting a shape similar to a gamma function and indicating little spatial or temporal variation of cloud optical thicknesses. Some distributions, e.g., for tropical regions with active deep convection (Figure 4: regions $1855,3019,3358,4186$ ), are multi-modal with both very thin and very thick clouds 
present, often at the same time. Some distributions, e.g., for tropical regions far from active convection (Figure 4: regions 1763 and 3272), are dominated by optically thin clouds. Some distributions, e.g., for midlatitude oceanic storm tracks (Figure 4: regions 717, 5677 and 5909), exhibit a very wide range of optical thickness values. Midlatitude land areas (Figure 4: regions 5387 and 6100 ) also have a very wide range of values but with more cirrus. This very large variety of cloud optical thickness distribution shapes presents a challenge to finding a simple representation of variability that is both general and accurate enough for calculating radiative fluxes.

Figure 5 translates the spatial variability of $\tau$ into distributions of $\epsilon$ and $\epsilon_{\mathbb{R}}$ and their respective average cloud albedo and emissivity biases, collected into three groups of small, moderate and large variability (Groups A, B and C, respectively). Table 1 cross-references the region numbers in Figures 3 and 4 with these groups. Clouds that exhibit relatively narrow optical thickness distributions also exhibit a narrow range of $\epsilon$ and $\epsilon_{\mathbb{R}}$, e.g., the marine boundary layer clouds (Group A). Note that the northern hemisphere regimes exhibit more variability because, at their higher latitude location, midlatitude disturbances occasionally enter the region bringing in optically thicker clouds (cf. Rozendaal and Rossow 2001). Some of the more complex distributions translate into a simple and broad distribution of $\epsilon$ but a more complex distribution of $\epsilon_{\mathrm{R}}$, e.g., mixtures of cirrus, mid-level clouds and boundary layer clouds that occur in "suppressed" convective or oceanic storm track regimes (Group B). Some groups show a very wide range of both $\epsilon$ and $\epsilon_{\mathbb{R}}$, e.g., active convection, especially over land, and midlatitude land areas (Group C). Convection in the South Pacific Convergence Zone exhibits a smoother distribution, dominated by moderate optical thicknesses, than the other convective regions. The oceanic storm track clouds tend to exhibit less overall variability that the clouds over midlatitude land areas; the latter exhibits much more frequent occurrence of isolated cirrus clouds associated with mountain ranges (Randall et al. 1996, Jin et al. 1996).

The distributions shown in Figure 4 are produced both by spatial and temporal variations 
of the clouds, whereas the distributions in Figure 5 represent the time variations of a spatial variation statistic. The effect we wish to focus on is the radiative effects of spatial variations of the clouds. However, some studies of the radiative effects of small-scale cloud variability have used time records of cloud variation taken at a point as an estimate of the cloud spatial variations (e.g., Cahalan et al. 1994b). Such an approach has to used carefully to be sure that the proper range of scales is included. Figure 6 shows the values of $\epsilon$ for six of the special study regions calculated from histograms of pixels aggregated over different combinations of space and time scales. Points on the $\mathrm{x}$-axis represent $\epsilon$ values produced solely by spatial variations at all scales below the marked value and points on the y-axis represent $\epsilon$ values produced solely by time variations at all scales below the marked value. Points in the interior of the figure are produced by both space and time variations at all scales smaller than the coordinate values of the point. This figure shows very clearly that the magnitude of the cloud variability statistic depends on the range of scales included: the larger the upper scale limit, the larger the variability. Moreover, the figure shows that the contribution from time variations generally grows more rapidly with scale than the contribution from spatial variations for most cloud regimes except for upper-level clouds over midlatitude oceans. The lower left panel in Figure 6 shows the results for the low-level clouds over ocean that have been used for almost all other studies of this problem: point-measurement records longer than about 10 days are equivalent to including spatial scales $>300 \mathrm{~km}$, which would overestimate the effects of cloud variability for typical model resolutions (Pincus et al. 1999 make this same point). In general (Figure 6), the purely spatial contributions to the cloud variations are much larger for upper-level clouds than lower-level clouds (left panels), for clouds over land than over ocean (center panels), and for clouds in the tropics than for clouds at midlatitudes (right panels). In the remainder of the paper, we examine cloud variations contributed solely by spatial scales $\leqslant$ $300 \mathrm{~km}$

Based on the pixel-level data from these sixteen regions, we construct the histogramaverage cloud albedos and emissivities, which are the "true" values (based on IPA), as well as the 
area-averaged quantities (at $280 \mathrm{~km}$ scale) at each time. Figure 7 shows the average cloud albedos and emissivities as functions of the linear-mean optical thickness, $\bar{\tau}$, with the homogeneous relation indicated by the dashed lines: significant deviations from the plane-parallel (i.e., homogeneous) relationships are apparent. Figures 8 and 9 show how the plane-parallel albedo and emissivity biases are related to three variability statistics: the optical thickness standard deviation, $\sigma_{\tau}$, the relative variance, $V=\left(\sigma_{\tau} / \bar{\tau}\right)^{2}$, and $\epsilon$ (also see Appendix). Although there is a general relationship between the first two statistics and the albedo error, $\epsilon$ provides a much more precise relationship (lower panel in Figure 8). Likewise $\epsilon_{\mathbb{R}}$ provides an improved relationship with the emissivity error, although not as good as for albedo error (lower panel in Figure 9).

Figure 10 examines the additional effect on longwave fluxes produced by small-scale variations of cloud top temperature (cf. Stubenrauch et al. 1999). The difference between the blackbody temperature representing the area-averaged longwave flux and the radiativelyweighted, area-averaged temperature reported in the ISCCP datasets exhibits very small errors for low-level and mid-level clouds; even for high-level clouds (lower panel in Figure 10), the averaging bias is $<1 \mathrm{~K}$, except for a very few cases where very cold and very warm clouds are mixed together. Even in these extreme cases, the flux error is only about $2-3 \%$.

\section{b. Relationship of shortwave and longwave inhomogeneity parameters}

In Section $2 \mathrm{e}$, we defined a quantity, $\epsilon_{\mathbb{R}}$, to estimate the emissivity of an inhomogeneous cloud from the mean properties and presented an empirical expression (given by Eqs. 16 and 17) to determine $\epsilon_{\mathrm{IR}}$ from $\bar{\tau}$ and $\epsilon$. This expression was developed using the pixel-level data from the sixteen regions. Figure 11 shows the scatterplot of $\epsilon_{\mathbb{R}}$ against $\epsilon$ for every region over the whole month, where deviations from the straight line representing equality increase as the average optical thickness increases. The curves illustrate the empirical fit used for Eqs. 16 and 17. Figure 
12a compares the calculated value, $\epsilon_{\mathbb{R}}$ ' against the true value, $\epsilon_{\mathbb{R}}$. The fit is generally very good, but there is increasing scatter and a slight overestimate for $\epsilon_{\mathbb{R}}>0.8$. Figure 5 shows that such large values of $\epsilon_{\mathrm{IR}}$ are very, very rare, occurring mostly in the active deep convection areas. Figure 12b shows that, despite the scatter exhibited in Figures 9 (lower panel) and 12a, the emissivities calculated with these relations generally agree well with the actual emissivities; the small population of underestimates by $0.1-0.2$ are produced in situations where the optical thickness distribution is multi-modal and there are mixtures of cirrus and deep convective clouds.

c. Relationship of inhomogeneity parameters to other cloud properties

We use the groupings, defined by the variability effects on cloud albedo and emissivity (Fig. 5 and Table 1) to look for correlations with other cloud properties that have also been shown to indicate different climate regimes (Rossow and Schiffer 1991, Rossow et al. 1993, Rossow and Schiffer 1999). Figure 13 shows the variations of $\epsilon$ and $\epsilon_{\mathbb{R}}$ with $\bar{\tau}$. Note that $\epsilon_{\mathbb{R}}$ is generally larger than $\epsilon$, usually because a small admixture of optically thinner clouds is always present with thicker clouds for which the emissivity is "saturated" at unity. The low variability Group A (upper panel in Figure 13), mainly boundary layer clouds over oceans, exhibits larger values of $\epsilon$ and $\epsilon_{\mathrm{IR}}$ only for intermediate optical thicknesses. Thus, the ISCCP cloud categories actually divide low-level clouds into three types: the small-mean optical thickness $(\hat{\tau} \leq 3.6)$ type with small-mean variability is called cumulus, the large-mean optical thickness $(\hat{\tau}>22.6)$ type with small-mean variability is called stratus. The middle-mean optical thickness type with largemean variability is called stratocumulus. Hahn et al. (2001) show that there is only a weak correspondence on average between these optical thickness categories and the classical morphological cloud types; but the results in Figure 13 suggest that the intermediate values of $\hat{\tau}$ are also systenatically associated with larger spatial variability at scales $<300 \mathrm{~km}$. Group B cases 
(center panel in Figure 13) are mixtures of clouds at all levels, found mostly in midlatitude storm track regions (and the northern hemisphere marine stratus regions that are occasionally influenced by midlatitude disturbances), that exhibit a general tendency for larger $\epsilon$ and $\epsilon_{\mathrm{IR}}$ as the average optical thickness increases. The analyses of Lau and Crane (1995) and Tselioudis et al. (2000) associate larger average optical thicknesses with stormier conditions and precipitation: such cloud systems produce a broad range of cloud properties. Group C (lower panel in Figure 13), containing mostly the tropical convective systems and the summertime midlatitude continents, exhibits a similar general increase of $\epsilon$ and $\epsilon_{\mathrm{RR}}$ with increasing optical thickness but with a larger range of values and much more scatter. This feature seems associated with even more extreme mixtures of slouds with very low optical thicknesses (cirrus) and very high optical thicknesses (deep convective clouds).

Figure 14 shows that there is no particular relationship between cloud fractional cover and the values of $\epsilon$ and $\epsilon_{\mathrm{RR}}$. This suggests that variations of cloud cover fraction have little to do with the magnitude of the cloud optical thickness spatial variations, which seem to be more indicative of cloud type (i.e., meteorology). Together with the weak relationship to $\bar{\tau}$, these results also confirm that $\epsilon$ and $\epsilon_{\mathbb{R}}$ provide independent information about clouds. 


\section{Climatology of Cloud Optical Parameter Variability}

a. Cloud optical thickness variability

Figure 15 shows maps of the 5-yr-mean (1986-1990 to avoid Pinatubo effects) values of $\epsilon$ for a region size of $280 \mathrm{~km}$ calculated from the ISCCP D1 dataset. Values are calculated separately for low-, middle- and high-level clouds (defined by cloud top pressures $\geq 680 \mathrm{mb}$, between 440 and $680 \mathrm{mb}$, and $<440 \mathrm{mb}$ ) and for all clouds together. Presented with cach map are normalized histograms of the mean $\epsilon$ values for land and ocean (with the global mean value indicated). Overall, these results show that high-level and mid-level clouds are more variable over land and low-level clouds are more variable over oceans. Consequently, clouds are slightly more variable over land than over ocean overall because of the preponderance of high- and middle-level clouds there. The geographic patterns of $\epsilon$ are fairly constant with season (hence only annual mean maps are shown), except for the well-known seasonal shifts of location of the InterTropical Convergence Zone (ITCZ) and northern hemisphere storm tracks (Rossow et al. 1993). Results for all twelve months (5-yr means) are available at the ISCCP Web site: http://isccp.giss.nasa.gov, given in terms of $\epsilon$ and $\epsilon_{\mathrm{IR}}$, as well as albedo and emissivity biases.

The geographic pattern of albedo bias produced by treating clouds as spatially homogeneous, even when weighted by mean cloud cover fraction, generally resembles the pattern of $\epsilon$ in Figure 15. Albedo errors are generally $<0.10$ for all clouds and $<0.05$ for individual cloud types. The largest errors occur where average cloud cover fractions are large, marking the ITCZ and midlatitude oceanic storm track regions.

For the individual cloud height types, the land/ocean histograms of $\epsilon$ are fairly similar in shape, where larger mean values are associated with the presence of relatively small populations of clouds with very large values of $\epsilon$ (also large values of $\bar{\tau}$, associated with precipitation). When all clouds are considered together, the distributions over land are bi-modal: most of the small $\epsilon$ values are found over North Africa. Generally, if $\epsilon$ is large for any cloud height category, 
then it is large for the combination of all clouds. The largest contribution to $\epsilon$ and the mean albedo bias in the ITCZ comes from the high-level clouds, whereas the middle- and low-level clouds predominate in the midlatitude oceanic storm track regions.

For high-level clouds (first panel in Figure 15), $\epsilon$ and the mean albedo bias form a fairly simple large-scale pattern composed of the ITCZ feature, the subtropical jet regions, the generation-portion of the midlatitude oceanic storm tracks, and clouds over major mountain ranges. The largest values of $\epsilon$ appear in the convective regions over land. Intermediate values of $\epsilon$ predominate over land, particularly adjacent to mountainous terrain. The most homogeneous high-level clouds are found over northern Africa and the Middle East and over the eastern margins of the ocean basins in the southern hemisphere. In such regions, the clouds tend to be optically very thin (i.e., cirrus) with small average cloud amounts (which ISCCP probably underestimates, cf. Liao et al. 1995, Jin et al. 1996).

Midule-level clouds (second panel in Figure 15) show a pattern of variability similar to high-level cloudiness, although there are significantly more large $\epsilon$ values at higher latitudes. The $\epsilon$ values in the polar regions over snow- and ice-covered surfaces are much less certain since the accuracy of the ISCCP optical thickness retrievals under these conditions is much lower (Rossow and Schiffer 1999). The concentration of large $\epsilon$ values near mountain ranges may be an extension of the high-level feature associated with frequent cirrus. The area of largest $\epsilon$ and albedo bias for middle-level clouds in the oceanic storm tracks is shifted down stream from the largest values for high-level clouds. Both the high latitude concentration and down-stream shift are even more apparent for low-level clouds. The variations of albedo bias are generally smaller because of the smaller cloud fractions of mid-level clouds.

Low-level clouds (third panel in Figure 15) exhibit some interesting, perhaps counterintuitive results. The global mean value of $\epsilon$ is about $11 \%$ larger over oceans than over land, but the mean albedo bias is fully $65 \%$ larger due to both a larger mean optical thickness and greater fraction of cloud cover of low-level clouds over oceans. The marine stratus regimes, off 
the subtropical west coasts of the continents, generally exhibit intermediate values of $\epsilon$ and albedo bias. This result contradicts the commonly held view that the thick stratus decks are the most homogeneous cloud type (as Cahalan 1989 also noted). While these clouds are statistically uniform over very large areas, they are the most variable low-level cloud type. Conversely, trade cumulus, usually thought to be highly inhomogeneous, are in fact, fairly homogeneous because their mean optical thicknesses are generally very small. The largest $\epsilon$ and albedo bias for low-level clouds in the oceanic storm tracks is shifted into the decay-portion of the tracks in the eastern oceanic basins. Most $\epsilon$ values over land are small to intermediate for low-level clouds and the albedo biases are uniformly small because of small average optical thicknesses and cloud cover fractions. In general, $\epsilon$ for low-level clouds depends primarily on surface type and does not exhibit the large-scale geographic features associated with the large-scale atmospheric circulation that show up in the distribution of middle- and high-level cloud $\epsilon$ values.

Figure 16 shows the mean seasonal variations of $\epsilon$ for high-, middle-, low-level and all clouds over ocean and land, averaged over the globe and in several climate zones: the tropics ( \pm $\left.15^{\circ}\right)$, midlatitudes $\left( \pm 30^{\circ}-60^{\circ}\right)$ and polar regions $\left( \pm 60^{\circ}-90^{\circ}\right)$. Monthly mean values were seasonally-phase-averaged for the period 1986-1990, e.g., values for January in the northern hemisphere are averaged with values for July in the southern hemisphere. The global mean $\epsilon$ values for high- and mid-level clouds are well correlated in their seasonal variations; they both exhibit a 12-month cycle that peaks in local mid-summer over land and in early autumn over oceans. Over land, low-level clouds show a 6 -month cycle with $\epsilon$-maxima in spring and autumn; over oceans there is a 12 -month cycle with its maximum in winter. The different seasonal behavior of low-level cloud variability suggests a difference in the underlying process that produces the variability (a similar contrast arises for the diurnal variations of clouds at different levels, Cairns 1995). A plausible explanation is that the behavior of low-level clouds is governed by seasonal changes in the boundary layer turbulence influenced strongly by the type of surface, whereas the high- and mid-level cloud variability is controlled by the characteristics of the large-scale 
atmospheric circulation regardless of surface type. The variability of high-level clouds in the tropics is larger than at other latitudes, whereas the variability of low-level clouds is largest in the polar regions. Middle-level clouds are most variable over polar oceans, but similar in variability in both the tropics and polar regions over land. The seasonal variations of high-level clouds are similar in phase in tropical and midlatitudes over oceans, but the polar clouds show a different seasonal phase with a maximum in winter (this may be spurious since ISCCP does not measure optical thicknesses in much of the winter polar regions, so these results may be caused by changing geographic coverage). Over land, the seasonal cycle of high-level clouds has the same phase at all latitudes. Low-level clouds also show similar seasonal cycles at all latitudes, but with maxima in winter over oceans and in spring and autumn over land. Middle-level clouds exhibit little seasonal variation in the tropics and midlatitudes over ocean, but track high-level cloud variations over land.

Combining these results with variations of monthly mean cloud cover (Rossow ef al. 1993 ) and other cloud properties (Rossow and Schiffer 1999), the classical climate regimes can be described by very different but essentially constant values of cloud properties, now extended to include the mesoscale variability of optical thickness. The convectively active regions of the tropics are characterized by extensive and persistent cloud cover with high-level tops (lower-level clouds are obscured in the satellite view), generally a mixture of optically very thin (cirrus) and very thick clouds (convective), resulting in a relative low mean optical thickness but very high optical thickness variability. The variation of cloud top temperatures in these regions is also relatively high (cf. Figure 17). The subtropics are characterized by low-level clouds, with much larger cloud cover, mean optical thickness and its variability in the eastern portions of the ocean basins than in the western portions. Midlatitudes exhibit extensive and persistent cover by very optically thick and variable clouds at all levels, especially over the oceans; the southern hemisphere seenis to exhibit less upper-level clouds whereas the more frequent appearance of cirrus over land increases the average optical thickness variability there. High latitudes also exhibit 
large cloud cover at mid- and low-levels with large average optical thicknesses, but the estimates of its variability are less reliable.

\section{b. Cloud emissivity and top temperature variability}

The cloud emissivity variation parameter, $\epsilon_{\mathbb{R}}$, is also reported at the above Web site for use in radiation codes that treat cloud effects in terms of albedo and emissivity (as opposed to calculations of both shortwave and longwave fluxes using a spectrally consistent optical thickness). This parameter represents only the variation of the longwave fluxes that occurs because of the small-scale variations of cloud optical thickness and does not account for the effect produced by a small-scale variations of cloud top temperature (cf. Stubenrauch et al. 1999). Since the cloud emissivity saturates at near-unity for relatively small optical thicknesses (see Figure 7), $\epsilon_{\mathbb{R}}$ is generally larger than $\epsilon$ (see Figure 11) because a small admixture of low optical thicknesses produces more emissivity variance than albedo variance. Nevertheless, this larger variability in $\epsilon_{\mathrm{IR}}$ translates into less effect on the emissivity because of the saturation effect. The close monotonic relationship between $\epsilon_{\mathbb{R}}$ and $\epsilon$ shown in Figure 11 implies that the geographic patterns of the two are very similar, and therefore, maps of $\epsilon_{\mathbb{R}}$ are not shown. The ratio of the effective infrared and solar wavelength optical thicknesses for inhomogeneous clouds generally decreases from unity at small optical thicknesses to about 0.2 at $\bar{\tau}=100$; however, the emissivity error made in assuming a homogeneous cloud is larger than the albedo error only for $\bar{\tau} \leqslant 15$.

The other factor contributing to variations of the longwave fluxes is variability of the cloud top temperature (likewise for cloud base effects on downwelling fluxes to the surface): if the cloud top temperature is treated as homogeneous at scales $\leqslant 300 \mathrm{~km}$ when it is variable, there will also be biases in the calculated longwave fluxes (cf. Stubenrauch et al. 1999), especially if the average temperature is calculated linearly. Figure 17 illustrates the average spatial standard deviation of cloud top temperature, $\sigma_{\mathrm{T}}$, from the ISCCP dataset: the larger this quantity, the 
larger the error in the calculated longwave flux obtained using the linear-average cloud top temperature. Figure 10 shows that the parameter reported in the ISCCP datasets, which is a radiatively-weighted-average cloud top temperature, represents the average longwave flux quite accurately with only a small underestimate for high clouds in cases of extreme variability, which occurs occasionally in the tropics. 


\section{Effects of Cloud Variability on the Radiation Budget}

To provide an estimate of the radiative effects of small-scale $(\leqslant 300 \mathrm{~km})$ cloud inhomogeneity, we calculate global shortwave and longwave fluxes at the top-of-atmosphere (TOA) and surface (SRF) for one particular day (15 July 1986) with and without accounting for the variability. We use the ISCCP dataset that is the same test case used by Zhang et al. (1995) to test parameter sensitivities in their flux calculations, so that the relative importance of cloud inhomogeneity can be judged against other factors affecting the radiation budget. The radiative transfer model used is essentially the same as they used. Table 2 summarizes the global mean effects on the shortwave and longwave fluxes of small-scale cloud inhomogeneity separated into two contributions. The first and largest contribution is the effect of using the radiative mean optical thicknesses, $\hat{\tau}$, in the calculations instead of the linear mean, $\bar{\tau}$, where the radiative mean value can be determined from the linear mean and $\epsilon$ using Eq. (10). Note the "average" optical thickness reported by ISCCP is $\hat{\tau}$. This larger effect $\left(15-20 \mathrm{Wm}^{-2}\right)$ would occur in a model only if the model produced the correct cloud water path values and used them directly to determine cloud radiative effects; in fact, most models have some adjustment to their parameterization to reduce this effect. The second and smaller contribution (almost $10 \mathrm{Wm}^{-2}$ ) accounts approximately for the 3-D effects by re-scaling the other optical parameters, $\varpi_{0}$ and $g$, using $\epsilon$ (cf. Cairns et al. 2000). The longwave effects shown do not account for cloud top temperature variability at smaller scales. The effects on shortwave fluxes are larger, about $20-30 \%$ of the average flux, whereas the longwave effects are only about $5 \%$ of the average flux. Figure 18 (lower panel) shows the zonal mean changes in the fluxes produced by the second part of the effect. Note the concentration of the shortwave changes in the ITCZ and midlatitude oceanic storm track zones (Figure 18, similar changes in downwelling flux at the surface are not shown), even though the largest total fluxes occur near the north pole (northern summer solar geometry). The changes in upwelling longwave fluxes at TOA are concentrated in the ITCZ (Figure 19, 
lower panel); changes in the downwelling longwave flux at the surface are relatively uniform with latitude. Both of these features are consistent with the regional distributions of the values of $\epsilon$ and $\epsilon_{\mathbb{R}}$

Stubenrauch et al. (1999) found that the observed relationship between shortwave and longwave fluxes for high-level clouds followed a curve that was more nearly linear than predicted by the theoretical relationship between optical thickness and emissivity. They showed that this behavior was consistent with the existence of mixtures of cloud top temperature within their 100 $\mathrm{km}$ scenes. We used a similar analysis to test the contribution to this behavior from the small-scale cloud optical thickness inhomogeneity effect and found that it has much less effect on this relationship than does the mixture of temperatures. This results from the fact that the larger inhomogeneity effects occur at larger cloud optical thickness values, producing noticeable changes in albedo but not in longwave emission because the emissivity has already reached unity. The key point from both of these studies is that the small-scale cloud variations alter the relationship between the shortwave and longwave flux effects in different ways for different types of clouds: the largest ratios of the emissivity bias to the albedo bias occurs for optical thicknesses between about 1 and 5 . As optical thicknesses increase above about 5 , the emissivity bias decreases as emissivities saturate at unity but the albedo bias continues to increase; as optical thicknesses decrease below 1, emissivity bias decreases more rapidly than the albedo bias (cf. Figure 7). These changes cannot be easily captured by parameterizations that relate cloud water directly to cloud albedo and emissivity. 


\section{Implications for General Circulation Modeling of Cloud Processes}

Dynamical considerations mitigate the effect that smaller-spatial-scale atmospheric (cloud) variations have on the overall transfer of energy to the atmosphere by radiation because the coupling of radiation to the cloud/atmosphere system also involves the time scale for radiative heating. Since the injection/loss of radiative energy into/from the atmosphere cannot occur on scales smaller than required to absorb shortwave/longwave photons, such coupling must occur at spatial scales larger than a few hundred meters. Since the major source of energy for the atmosphere is convective (turbulent) transports from the solar-heated surface, in both sensible and latent forms, at spatial scales ranging from the depth of the planetary boundary layer $(\sim 1-3 \mathrm{~km})$ to the depth of the whole troposphere $(\sim 10-15 \mathrm{~km})$, the heating effect of these motions requires aggregation of the convective variability over time scales of order ten times the eddy overturning times, $\sim 1-3$ hrs. Thus, differential heating of cloud elements at spatial scales smaller than a few kilometers and time scales less than about an hour can have little influence on the aggregate heat flux across the atmosphere. Also, since atmospheric motions at these small scales are predominantly three-dimensional (cf. Lilly 1983), turbulent energy cascade will efficiently dissipate any differential radiative heating at scales of a few kilometers on time scales $\sim 10 \mathrm{~min}$, long before they could be transported very far by the larger-scale motions. Thus, the main effect of the smaller scale variability of the atmosphere is its systematic alteration of the relationships between the radiative fluxes and mean atmospheric optical properties.

For a GCM that determines the gridbox (i.e., linear) mean cloud water content, together with the microphysical parameters, the vertical layer structure and the cloud cover fraction, the homogeneous optical thickness, single scattering albedo and asymmetry parameter can be calculated. Then the climatology of $\epsilon$ (and/or $\epsilon_{\mathbb{R}}$ ) can be used to re-scale these homogeneous parameters to account statistically for the effects of the smaller-scale cloud variations. The values of $\epsilon$ (and $\epsilon_{\mathbb{R}}$ ) for other spatial resolutions than the $280 \mathrm{~km}$ domain used here can be estimated by assuming that these values scale roughly linearly with area as our analysis shows. 
The evolution of the fidelity of the representation of a physical process in a GCM usually involves three stages: first, some parameters are specified (either constant or with "climatological" variations), then improvements are introduced where these parameters are diagnosed from the prognostic variables, and finally further improvements lead to making these parameters prognostic variables. The model "improvement" that we are proposing augments the usual "large-scale" cloud parameters (cloud cover fraction, optical thickness, temperature, vertical structure, particle phase, shape and size distribution) needed for describing their effects on radiative fluxes by adding a measure of the mesoscale variability of cloud optical thickness (also small-scale cloud top temperature variability). We consider all of these parameters to be "large-scale" in the sense that they are varied explicitly to represent the synoptic (cloud type) variations of the cloud radiative properties at all other scales larger than that of the model map grid. The results reported here (and the datasets from the Web site) can then be used to specify mesoscale cloud variations as a function of cloud height, location and month-of-year, to search for diagnostic relationships with meteorological conditions, and to verify a prognostic model of small-scale variability. 


\section{APPENDIX}

a. Statistical issues

A number of papers have considered the parameterization of statistical distributions of cloud optical thickness using analytical probability distribution functions. The gamma distribution has been most extensively investigated, probably because it can be analytically integrated over the two-stream solution of the radiative transfer equation (Barker et al. 1996, Barker and Wielicki 1997). The log normal distribution has also been found to be a useful approximation to liquid water path statistics in the analysis of large scale datasets such as those analyzed here (Cahalan ef al. 1994a, Oreopoulos and Davies 1998b). In general if we measure the mean of two functions of a parameter [e.g., $\overline{f(\tau)}$ and $\overline{g(\tau)}$ ], then the Maximum Entropy (ME) distribution associated with these measurements is

$$
p(\tau ; a, b)=N \exp [a f(\tau)+b g(\tau)]
$$

where $\mathrm{N}$ is the total population size and the constants, $\mathrm{a}$ and $\mathrm{b}$, can be estimated from $\overline{f(\tau)}$ and

$\overline{g(\tau)}$. The gamma distribution and the log normal distribution are the ME distributions

associated with $[f(\tau)=\tau, g(\tau)=\ln (\tau)]_{\text {and }}\left[f(\tau)=\ln (\tau), g(\tau)=\sigma_{\ln (\tau)}\right]$, respectively. A desirable aspect of the ME formalism is that the estimates of $a$ and $b$ are guaranteed to be sufficient statistics, as opposed to using the method of moments (MOM), which has been found to be problematic (Oreopoulos and Davies 1998b). Using this formalism also allows us to introduce a generalized probability distribution function 


$$
p(\tau ; a, b)=N \exp [a * \tau+b * R(\tau)]
$$

where $R(\tau)$ is the function relating albedo and optical thickness, that can be determined from the mean optical thickness and albedo. This distribution guarantees that there is no optical thickness or albedo bias by definition. This type of function can be estimated directly from the ISCCP dataset which reports both $\bar{\tau}$ and $\hat{\tau}$, the latter of which is proportional to albedo. It could also be estimated from data at coarse spatial resolution if albedo and liquid water path are measured, for example, from CERES and SMM/I. If the cloud albedo were strictly a logarithmic function of optical thickness, this distribution would reduce to the gamma distribution; however, cloud albedo depends on more parameters than optical thickness.

b. Discrete gamma distribution approximation

The gamma distribution (e.g., Barker et al. 1996) of $\tau_{i}$ (where the subscript indicates pixel number) may be expressed as

$$
p\left(\tau_{i} ; \bar{\tau}, v\right)=\left[\tau_{i} \Gamma(v)\right]^{-1}\left(\frac{v \tau_{i}}{\bar{\tau}}\right)^{v} \exp \left(\frac{-v \tau_{i}}{\bar{\tau}}\right)
$$

$$
\text { for } \tau_{i}>0
$$

Here $\Gamma(v)$ is the gamma function and $v$ is the shape parameter that represents the variability of the distribution. In addition to the standard deviation and the linear and radiatively-weighted mean values of cloud optical thickness, the ISCCP D1 dataset also reports a six-interval distribution of optical thicknesses that represent approximately equal intervals of cloud albedo. Thus, another 
way to represent the smaller-scale cloud optical thickness variability would be to determine a gamma function fit to these distributions. To be consistent with Barker et al. (1996) in this illustration, the histograms are truncated to the same range of optical thicknesses and then normalized to account for the truncated populations (which is particularly important for distributions with $v<2$ ). The "true" albedo is determined directly from the radiatively-weighted average optical thickness; the linear average and standard deviation are also calculated from these histograms. All that remains is to determine the appropriate shape parameter, $v$.

We compare different approaches for estimating $v$ from the ISCCP dataset. A common method is the method of moments

MOM:

$$
\bar{v}=\left(\frac{\bar{\tau}}{\sigma_{\tau}}\right)^{2}=V^{-2}
$$

where $\mathrm{V}$ is the relative variance of the distribution (or dispersion). This estimate cannot be guaranteed in the sense of having reliable confidence intervals, but it has been found to work where the distributions are unimodal and "smooth" (Newman et al. 1995). For less well-behaved distributions, it does not work well (Oreopoulos and Davies 1998b). In our global study, we frequently encountered ill-behaved optical thickness distributions (cf. Fig. 4) and, therefore, sought a more robust determination of the gamma distribution that could be applied to satellite data with coarser resolution (i.e., smaller sample populations). We have employed the Maximum Likelihood Estimate (MLE) to determine $v$ using the expression

MLE:

$$
\tilde{v}^{-1} \exp [\Psi(\tilde{v})]=\bar{\tau}^{-1} \exp [\overline{\ln (\tau)}]
$$


where $\Psi$ is the digamma function and $\tilde{v}$ is the logarithmic average of $v$. The MLE for $v$ is only asymptotically unbiased, similar to the MLE estimate of the variance, so care should be exercised if sample populations are very small. This problem can be corrected by using the ME method, which also provides confidence intervals for $v$. We use an approximation to (A5) because ISCCP reports the value of $\hat{\tau}$ instead of $\tilde{\tau}$ :

$$
\bar{\tau}^{-1} \exp [\overline{\ln (\tau)}] \approx \bar{\tau}^{-1} R^{-1}[\overline{R(\tau)}]=\frac{\hat{\tau}}{\bar{\tau}}=(1-\varepsilon)
$$

so that

$$
\tilde{v}^{-1} \exp [\Psi(\tilde{v})] \approx(1-\varepsilon)
$$

This expression introduces an explicit relationship between the inhomogeneity parameter computed for a cloudy scene and the gamma distribution used to model it. For the range of $\epsilon$ encountered, (A7) may be approximated using

$$
\hat{v}=[\varepsilon-\ln (1-\varepsilon)]^{-1}
$$

with an rms error of $\sim 0.6 \%$ for $0.01 \leq \epsilon \leq 0.8$. To improve (A8), sample gamma distributions were generated for a range of $v$ and $\bar{\tau}$ values and the resulting $\epsilon$ values computed. From this exercise it was determined that (A8) could be modified to give a better approximation to the 
correct (as specified) shape parameter as follows:

$$
v^{\prime}=\hat{v} \sqrt{\frac{\hat{\tau}}{\tau_{0}}}=[\varepsilon-\ln (1-\varepsilon)]^{-i} \sqrt{\frac{(1-\varepsilon) \bar{\tau}}{\tau_{0}}}
$$

Quasi-MLE:

where $\tau_{0}$ is a reference optical thickness value $(=15)$. The largest remaining error in using (A9) is an overestimation of large $v$ values; but, since these distributions represent homogeneous situations, the radiative significance of this error is slight.

b. Evaluation of gamma distribution representations at $3 \mathrm{hr}, 280 \mathrm{~km}$ scale

The relative success of the three gamma distribution fits (MOM, MLE and Quasi-MLE) in modeling the observed cloud fields was judged radiatively, i.e., by how well each fit reproduced the albedo of the original clouds. Plane-parallel (homogeneous) albedo computations were also included for comparison. Note that here we use the gamma function fits to calculate albedo even though it can be obtained directly from the radiatively-weighted mean optical thickness as we have shown; however, such analytic functions might be useful in GCM parameterizations.

Results using these three fits for the three groups of ISCCP DX data used in Section 3 are shown in Figure $A 1$ as an absolute albedo error, $A_{i}-A_{\text {this, }}$ versus $\epsilon$. Fractional errors (normalized by the histogram-average albedo, $A_{\text {hiss }}$ ) were avoided so as not to over-emphasize small absolute errors. Results were also not scaled by cloud amount, so they represent a "worst-case" error. The systematic overestimation inherent in the plane-parallel assumption is clear in Figure Al (upper left panel), as is the considerable improvement gained by using any of the gamma-distribution models. For group A $(\epsilon \leq 0.1)$, the average over-estimate of cloud albedo using the plane-parallel 
method is about 0.012 . The MOM and MLE methods reduce the average albedo error to about 0.002; the Quasi-MLE method reduces the error further to about 0.0004 . Thus, all three methods reduce the error substantially for homogeneous clouds as expected, since in this case the optical thickness distributions are nearly Gaussian in shape with small standard deviations.

For moderately inhomogeneous clouds (group B, $0.1<\epsilon \leq 0.2$ ), the average albedo error of MOM is slightly smaller than for MLE and quasi-MLE; but all three methods reduce the plane-parallel bias by more than $90 \%$. The Quasi-MLE method shows much less scatter than the other two methods. For $\epsilon>0.2$, the mean error of the MOM decreases more than for the other two, but the scatter becomes much more pronounced, especially at moderate-to-high values of $\epsilon$, because of the use of the variance in the computation of $v$. For unimodal distributions, this is not a problem; but for mixtures of optically thin cirrus and optically thick convective clouds, common in the tropics, this method can either overweight the thicker clouds through their effect on the variance, when the mean value is small, or the thinner clouds when the mean value is larger. The MLE method is biased increasingly high as $\epsilon$ increases and exhibits significant scatter as well because of the use of the logarithm as an approximation to the radiative weighting in determining $v$. The error is be largest for distributions with $\bar{\tau}<2$ or $\bar{\tau}>40$. The Quasi-MLE method does better but still shows a weak trend with $\epsilon$, indicating that the scaling used in (A7) does not fully account for the observed variations of $\epsilon$ with $\bar{v}$. All three methods still reduce the plane-parallel bias error by more than $98 \%$. 


\section{References}

Astin, I., and L. Di Girolamo, 1999: A general formalism for the distribution of the total length of a geophysical parameter along a finite transect. IEEE Trans. Geosci. Rem. Sens., 37, 508512 .

Barker, H.W., 1992: Solar radiative transfer through clouds possessing isotropic variable extinction coefficient. Quart. J. Roy. Meteor. Soc., 118, 1145-1162.

Barker, H.W., 1995: A spectral analysis of albedo and bidirectional reflectances for inhomogeneous clouds. Remote Sens. Environ., 54, 113-120.

Barker, H.W., 1996a: A parameterization for computing grid-averaged solar fluxes for inhomogeneous marine boundary layer clouds. Part I: Methodology and homogeneous biases. J. Atmos. Sci., 53, 2289-2303.

Barker, H.W., 1996b: Estimating cloud field albedo using one-dimensional series of optical depth. J. Atmos. Sci., 53, 2826-2837.

Barker, H.W., and J.A. Davies, 1992: Solar radiative fluxes for stochastic, scale-invariant broken cloud fields. J. Atmos. Sci. 49, 1115-1125.

Barker, H.W., and Q. Fu, 1999: Modelling domain-averaged solar fluxes for an evolving tropical cloud system. Atmos. Oceanic Opt., 12, 211-217.

Barker, H.W., and D. Liu, 1995: Inferring optical depth of broken clouds from Landsat data. J. Climate, 8, 2620-2630.

Barker, H.W., and B.A. Wielicki, 1997: Parameterizing grid-averaged longwave fluxes for inhomogeneous marine boundary layer clouds. J. Atmos. Sci., 54, 2785-2798.

Barker, H.W., B.A. Wielicki and L. Parker, 1996: A parameterization for computing gridaveraged solar fluxes for inhomogeneous marine boundary layer clouds. Part II: Validation using satellite data. J. Atmos. Sci., 53, 2304-2316.

Barker, H.W., G.L. Stephens and Q, Fu, 1999: The sensitivity of domain-averaged solar fluxes to 
assumptions about cloud geometry. Quart. J. Roy. Meteor. Soc., 125, 2127-2152.

Byrne, R.N., R.C.J. Somerville and B. Subasilar, 1996: Broken-cloud enhancement of solar radiation absorption. J. Atmos. Sci., 53, 878-886.

Cahalan, R.F., 1989: Overview of fractal clouds. Advances in Remote Sensing. A Deepak, Ed., A. Deepak, 317-389.

Cahalan, R.F., W. Ridgway, W.J. Wiscombe, T.L. Bell and J.B. Snider, 1994a: The albedo of fractal stratocumulus clouds. J. Atmos. Sci., 51, 2434-2455.

Cahalan, R.F., W. Ridgway, W.J. Wiscombe, S. Gollmer and Harshvardhan, 1994b: Independent pixel and Monte Carlo estimates of stratocumulus albedo. J. Atmos. Sci., 51, 3776-3790.

Cahalan, R.F., D. Silberstein and J.B. Snider, 1995: Liquid water path and plane-parallel albedo bias during ASTEX. J. Atmos. Sci., 52, 3002-3012.

Cairns, B., A.A. Lacis and B.E. Carlson, 2000: Absorption within inhomogeneous clouds and its parameterization in general circulation models. J. Atmos. Sci., 57, 700-714.

Chambers, L.H., B.A. Wielicki and K.F. Evans, 1997a: Accuracy of the independent pixel approximation for satellite estimates of oceanic boundary layer cloud optical depth. $J$. Geophys. Res., 102, 1779-1794.

Chambers, L.H., B.A. Wielicki and K.F. Evans, 1997b: Independent pixel and two-dimensional estimates of Landsat-derived cloud field albedo. J. Atmos. Sci., 54, 1525-1532.

Coley, P.F., and P.R. Jonas, 1996: The influence of cloud structure and droplet concentration on the reflectance of shortwave radiation. Ann. Geophysicae, 14, 845-852.

Considine, G., J.A. Curry and B.A. Wielicki, 1997: Modeling cloud fraction and horizontal variability in marine boundary layer clouds. J. Geophys. Res., 102, 13,517-13,525.

Davies, R., 1978: The effect of finite geometry on the three-dimensional transfer of solar irradiance in clouds. J. Atmos. Sci., 35, 1712-1725.

Davis, A., A. Marshak, W. Wiscombe and R. Cahalan, 1996: Scale invariance of liquid water distributions in marine stratocumulus, Part I: Spectral properties and stationarity issues. $J$. 
Atmos. Sci. 53, 1538-1558.

Davis, A., A. Marshak, R. Cahalan and W. Wiscombe, 1997: The Landsat scale break in stratocumulus as a three-dimensional radiative transfer effect: Implications for cloud remote sensing. J. Atmos. Sci., 54, 241-260.

Davis, A., A. Marshak, H. Gerber and W.J. Wiscombe, 1999: Horizontal structure of marine boundary layer clouds from centimeter to kilometer scales. J. Geophys. Res., 104, 61236144 .

Del Genio, A.D., M-S. Yao, W. Kovari and K-W Lo, 1996: A prognostic cloud water parameterization for global climate models. J. Climate, 9, 270-303.

DiGirolamo, L., R. Davies, 1997: Cloud fraction errors caused by finite resolution measurements. J. Geophys. Res., 102, 1739-1756.

Fowler, L.D., D.A. Randall and S.A. Rutledge, 1996: Liquid and ice cloud microphysics in the CSU general circulation model. I: Model description and simulated microphysical processes. J. Climate, 9, 489-529.

Goody, R.M., and Y.L. Yung: 1989: Atmospheric Radiation: Theoretical Basis. Oxford, New York, pp519.

Hahn, C.J., W.B. Rossow and S.G. Warren, 2001: ISCCP cloud properties associated with standard cloud types identified in individual surface observations. J. Climate, 14, 11-28

Hansen, J.E., and L.D. Travis, 1974: Light scattering in planetary atmospheres. Space Sci. Rev., $16,527-610$.

Hignett, P., 1987: A study of the short-wave radiative properties of marine stratus: Aircraft measurements and model comparisons. Quart. J. Roy. Meteor. Soc., 113, 1011-1024.

Jaynes, E.T., 1968: Prior probabilities. IEEE Trans. Sys. Sci. Cyb., 4, 227-241.

Jin, Y., W.B. Rossow and D.P. Wylie, 1996: Comparison of the climatologies of high-level clouds from HIRS and ISCCP. J. Climate, 9, 2850-2879.

Jonas, P.J., 1994: Turbulence and cloud microphysics. Atmos. Res., 40, 283-306. 
Kobayashi, T., 1991 : Reflected solar flux for horizontally inhomogeneous atmospheres. J. Atmos. Sci., 48, 2436-2447.

Kobayashi, T., 1993: Effects due to cloud geometry on biases in the albedo derived from radiance measurements. J. Climate, 6, 120-128.

Kobayashi, T., K. Masuda and A. Uichiyama, 1999: Enhanced visible radiance near clouds in the OCTS measurements. Atmos. Oceanic Opt., 12, 218-221.

Kobayashi, T., K. Masuda, M. Sasaki and J. Mueller, 2000: Monte Carlo simulations of enhanced visible radiance in clear-air satellite fields of view near clouds. J. Geophys. Res., (in press).

Korolev, A.V., and I.P. Mazin, 1993: Zones of increased and decreased droplet concentration in stratiform clouds. J. Appl. Meteor., 32, 760-773.

Lacis, A.A., and V. Oinas, 1997: A description of the correlated k distribution method for modeling nongray gaseous absorption, thermal emission, and multiple scattering in vertically in homogeneous atmospheres. J. Geophys. Res., 96, 9027-9063.

Lau, N-C., and M.W. Crane, 1995: A satellite view of the synoptic-scale organization of cloud properties in midlatitude and tropical circulation systems. Mon. Wea. Rev., 123, 19842006.

Lenschow, D.H., and B.B. Stankov, 1986: Length scales in the convective boundary layer. $J$. Atmos. Sci. , 43, 1198-1209.

Li, J., D.J. Geldart and P. Chylek, 1994: Solar radiative transfer in clouds with vertical internal inhomogeneity. J. Atmos. Sci., 51, 2542-2552.

Liao, X., W.B. Rossow and D. Rind, 1995: Comparison between SAGE II and ISCCP high-level cloud amounts. J. Geophys. Res., 100, 1121-1135.

Lilly, D.K., 1983: Stratified turbulence and the mesoscale variability of the atmosphere. J. Atmos. Sci., 40, 749-751.

Lilly, D.K., and E.L. Petersen, 1983: Aircraft measurements of atmospheric kinetic energy spectra. Tellus, 35A, 379-382. 
Loeb, N.G., and J.A. Coakley, 1998: Inference of marine stratus cloud optical depths from satellite measurements: Does 1D theory apply? J. Climate, 11, 215-233.

Loeb, N.G., and R. Davies, 1997: Angular dependence of observed reflectances: A comparison with plane parallel theory. J. Geophys. Res., 102, 6865-6881.

Lovejoy, S., and D. Schertzer, 1990: Multi-fractals, universality classes and satellite and radar measurements of clouds and rain fields. J. Geophys. Res., 95, 2021-2034.

Lovejoy, S., D. Schertzer, P. Silas, Y. Tessier and D. Lavallee, 1993: The unified scaling model of atmospheric dynamics and systematic analysis of scale invariance in cloud radiances. Ann. Geophys., 11, 119-127.

Marshak, A., A. Davis, W. Wiscombe and R. Cahalan, 1995: Radiative smoothing in fractal clouds. J. Geophys. Res., 100, 26,247-26,261.

Marshak, A., A. Davis, W.J. Wiscombe and R.F. Cahalan, 1997: Scale invariance of liquid water distributions in marine stratocumulus, Part II: Multifractal properties and intermittancy issues. J. Atmos. Sci., 54, 1423-1444.

Marshak, A., A. Davis, W. Wiscombe, W. Ridgway and R. Cahalan, 1998a: Biases in shortwave column absorption in the presence of fractal clouds. J. Climate, 11, 431-446.

Marshak, A., A. Davis, W. Wiscombe and R. Cahalan, 1998b: Radiative effects of sub-mean free path liquid water variability observed in stratiform clouds. J. Geophys. Res., 103, 19,55719,567 .

Nair, U.S., R.C. Weger, K.S. Kuo and R.M. Welch, 1998: Clustering, randomness, and regularity in cloud fields. 5. The nature of regular cumulus cloud fields. J. Geophys. Res., 103, $11,363-11,380$

Newman, W.I., J.K. Lew, G.L. Siscoe and R.G. Fovell, 1995: Systematic effects of randomness in radiative transfer. J. Atmos. Sci., 52, 427-435.

Oreopoulos, L., and H.W. Barker, 1999: Accounting for subgrid-scale cloud variability in a multilayer 1D solar radiative transfer algorithm. Quart. J. Roy. Meteor. Soc., 125, 301-330. 
Oreopoulos, L., and R. Davies, 1998a: Plane parallel albedo biases from satellite observations.

Part I: Dependence on resolution and other factors. J. Climate, 11, 919-932.

Oreopoulos, L., and R. Davies, 1998b: Plane parallel albedo biases from satellite observations.

Part II: Parameterizations for bias removal. J. Climate, 11, 933-944.

Pincus, R., S.A. McFarlane and S.A. Klein, 1999: Albedo bias and the horizontal variability of clouds in subtropical marine boundary layers: Observations from ships and satellites. $J$. Geophys. Res., 104, 6183-6191.

Ramirez, J.A., and R.L. Bras, 1990: Clustered or random cloud fields: The statistical character of observed and simulated cloud fields. J. Geophys. Res., 95, 2047-2059.

Randall, D.A., B. Albrecht, S. Cox, D. Johnson, P. Minnis, W. Rossow and D. O'C. Starr, 1996: On FIRE at ten. Adv. Geophys., 38, 37-177.

Rossow, W.B., 1989: Measuring cloud properties from space: A review. J. Climate, 2, $201-213$.

Rossow, W B., and B. Cairns, 1995: Monitoring changes of clouds. Climate Change, 31, 305347.

Rossow, W.B., and R.A. Schiffer, 1991: ISCCP cloud data products. Bull. Amer. Meteor. Soc., $72,2-20$.

Rossow, W.B., and R.A. Schiffer, 1999: Advances in understanding clouds from ISCCP. Bull. Amer. Meteor. Soc., 80, 2261-2287.

Rossow, W.B., A.W. Walker and L.C. Garder, 1993: Comparison of ISCCP and other cloud amounts. J. Climate, 6, 2394-2418.

Rossow, W.B., A.W. Walker, D. Beuschel and M. Roiter, 1996: International Satellite Cloud Climatology (ISCCP) description of new cloud datsets. WMO/TD 737, World Climate Research Programme (ICSU and WMO), 115pp.

Rozendaal, M., and W.B. Rossow, 2001: Characterizing the influence of the general circulation on marine boundary layer clouds. J. Atmos. Sci., (submitted).

Seze, G., and W.B. Rossow, 1991a: Time-cumulated visible and infrared radiance histograms 
used as descriptors of surface and cloud variations. Int. J. Remote Sensing, 12, 877-920.

Seze, G., and W.B. Rossow, 1991b: Effects of satellite data resolution on measuring the space/time variations of surfaces and clouds. Int. J. Remote Sensing, 12, 921-952.

Smith, R.N.B., 1990: A scheme for predicting layer clouds and their water content in a general circulation model. Quart. J. Roy. Meteor. Soc., 116, 435-460.

Stephens, G.L., 1978: Radiation profiles in extended water clouds. II: Parameterization schemes. J. Atmos. Sci., 35, 2123-2132.

Stephens, G.L., 1988a: Radiative transfer through arbitrarily shaped optical media, Part I: A general method of solution. J. Atmos. Sci., 45, 1818-1836.

Stephens, G.L., 1988b: Radiative transfer through arbitrarily shaped optical media, Part II: Group theory and simple closures. J. Atmos. Sci., 45, 1837-1848.

Stubenrauch, C.J., W.B. Rossow, N.A. Scott and A. Chedin, 1999: Clouds as seen by satellite sounders (3I) and imagers (ISCCP). Part III: Combining 3I and ISCCP cloud parameters for better understanding of cloud radiative effects. J. Climate, 12, 3419-3442.

Sundqvist, H., 1978: A parameterization scheme for non-convective condensation including prediction of cloud water content. Quart. J. Roy. Meteor. Soc., 104, 677-690.

Tiedtke, M., 1993: Representation of clouds in large-scale models. Mon. Wea. Rev., 121, 30403061 .

Tiedtke, M., 1996: An extension of cloud-radiation parameterization in the ECMWF model: The representation of subgrid-scale variations of optical depth. Mon. Wea. Rev., 124, 745-750.

Tselioudis, G., Y-C. Zhang and W.B. Rossow, 2000: Cloud and radiation variations associated with northern midlatitude low and high sea level pressure regimes. J. Climate, 13, 312 327 .

Wang, J-H., W.B. Rossow and Y-C. Zhang, 2000: Cloud vertical structure and its variations from a 20-yr global rawinsonde dataset. J. Climate, 13, 3041-3056.

Wielicki, B.A., and L. Parker, 1992: On the determination of cloud cover from satellite sensors: 
The effect of sensor spatial resolution. J. Geophys. Res., 97, 12,799-12,823.

Zhang, Y-C., W.B. Rossow and A.A. Lacis, 1995: Calculation of surface and top-of-atmosphere radiative fluxes from physical quantities based on ISCCP datasets, Part I: Method and sensitivity to input data uncertainties. J. Geophys. Res., 100, 1149-1165.

Zuev, V.E., and G.A. Titov, 1995: Radiative transfer in cloud fields with random geometry. $J$. Atmos. Sci., 52, 176-190. 


\section{Tables}

Table 1. Cloudy regions grouped by the magnitude of their small-scale variations.

Group A Group B Group C

Marine Boundary Layer $\quad 2072,2446,2690 \quad 4647,4940$

Active Tropical Convection

1855

$3019,3358,4186$

Tropical Cirrus

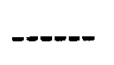

1763

3272

Ocean Storm Tracks

$---$

$717,5677,5909$

Midlatitude Land

-----

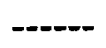

5387,6100 
Table 2. Changes in global, daily mean, upwelling ( $\uparrow$ ) and downwelling ( $($ ), shortwave (SW) and longwave (LW) fluxes $\left(\mathrm{Wm}^{-2}\right)$ at the top-of-atmosphere (TOA) and surface (SRF) produced by re-scaling only the linear mean optical thickness using $\epsilon$ and by re-scaling all optical parameters using $\epsilon$. Values are shown for overcast conditions and scaled by cloud cover fraction (CF). Results are based on a test calculation using ISCCP data for one particular day, 15 July 1986.

\begin{tabular}{llcc}
\hline & $\langle\tau\rangle-\left\langle\tau^{\prime}\right\rangle$ only & $\varpi_{0}, g \rightarrow \varpi_{0}{ }^{\prime}, g^{\prime}$ & Total \\
\hline TOA-SW $\uparrow$ & -15 & -7 & -22 \\
CF*TOA-SW $\uparrow$ & -13 & -6 & -19 \\
SRF-SW! & +19 & +8 & +27 \\
CF*SRF-SW! & +15 & +7 & +22 \\
\hline TOA-LW 1 & +6 & +3 & +9 \\
CF*TOA-LW 1 & +4 & +3 & +7 \\
SRF-LW! & -6 & -3 & -6 \\
CF*SRF-LW! & -4 & -2 & 6 \\
\hline
\end{tabular}




\section{Figure Captions}

Figure 1: Schematic illustrating different assumptions about variations of optical media used to model radiative transfer through cloudy atmospheres: (a) horizontally homogeneous layers with properties that vary only in the vertical, (b) horizontally and vertically inhomgeneous layer, (c) horizontally and vertically inhomogeneous layer that is statistically homogeneous in the horizontal direction.

Figure 2: Geometry for calculating the contribution of each element of the Earth's surface area to the upwelling flux observed at height, $h$, above the surface, where $R$ is the radius of the Earth, $r$ is the radius of an annular surface region at central angle, $\varphi$, width, $\Delta \varphi$, and distance, $\mathrm{d}$, from the observation point. The angle, $\theta$, is the viewing-zenith angle at the center of the annular region.

Figure 3: Locations of special regions in the viewing area of each geostationary satellite that were selected for detailed study.

Figure 4: Distributions of visible optical thicknesses for each of the special study regions (Figure 3) aggregated over the month of July 1992.

Figure 5: Distributions of $\epsilon$ and $\epsilon_{\mathbb{R}}$ values for the special study regions (Figure 3) aggregated over the month of July 1992 and collected into three groups according to their magnitude (Tab!e 1). These statistics are calculated for areas of about $(280 \mathrm{~km})^{2}$.

Figure 6: Increase of $\epsilon$ with increasing time (logarithm days) and space (logarithm kilometers) scales for regions selected to represent general cloud type regimes: middle- and high-level clouds (upper left panel), low-level clouds (lower left panel), midlatitude low-level clouds over land (upper central panel), midlatitude low-level clouds over ocean (lower central panel), midlatitude 
middle- and high-level clouds over ocean (upper right panel) and tropical middle- and high-level clouds over oceans (lower right panel). Values on y-axis represent time variation contributions alone; values on the $\mathrm{x}$-axis represent space variation contributions alone. Values in the interior represent combinations of space and time variation contributions. Box numbers refer to Figure 3 and Table 1.

Figure 7: Cloud albedo (upper panel) and emissivity (lower panel) as a function of the linear-areamean optical thickness, ${ }^{\bar{\tau}}$, in all the special study regions: the dashed line represents the relationship for a homogeneous plane-parallel cloud layer (two different lines are given for emissivity depending on whether the cloud is liquid or ice).

Figure 8: Plane-parallel albedo bias plotted as function of the standard deviation of optical thicknesses (upper panel), the relative variance of optical thicknesses (center panel), and the parameter $\epsilon$ (lower panel) at a scale of about $300 \mathrm{~km}$ for all special study areas.

Figure 9: Plane-parallel emissivity bias plotted as function of the standard deviation of optical thicknesses (upper panel), the relative variance of optical thicknesses (center panel), and the parameter $\epsilon_{\mathrm{IR}}$ (lower panel) at a scale of about $300 \mathrm{~km}$ for all special study areas.

Figure 10: Difference between the average longwave flux, represented by the average of the fourth power of the emission temperature, and the average cloud top temperature reported in ISCCP datasets as function of the area standard deviation of cloud top temperatures, $\sigma_{\mathrm{T}}$, for all clouds (upper panel) and for high-level clouds only (lower panel).

Figure 11: Observed relationship of $\epsilon_{\mathbb{R}}$ and $\epsilon$ for all special study areas. Curves are for circles 
with different radii that are functions of optical thickness that all pass through the points $(0,0)$ and $(1,1)$.

Figure 12a: Estimated values, $\epsilon_{\mathbb{R}}^{\prime}$, versus the true values, $\epsilon_{\mathbb{R}}$. The estimated values are calculated from an empirical relation with $\bar{\tau}$ and $\epsilon$.

Figure $12 \mathrm{~b}$ : Estimated values of emissivity, E', versus the true values, E. The estimated values are calculated from an empirical relation with $\epsilon_{\mathbb{I R}}^{\prime}$.

Figure 13: Distributions of $\epsilon$ (closed circles) and $\epsilon_{\mathrm{IR}}$ (open circles) as functions of the linear mean optical thickness for the three groups of special study regions (Table 1).

Figure 14: Distributions of $\epsilon$ (closed circles) and $\epsilon_{\mathbb{R}}$ (open circles) as functions of cloud cover fraction for the three groups of special study regions (Table 1).

Figure 15: Geographic distribution of the annual mean values of $\epsilon$, averaged over 1986-1990, for high-, mid-, low-level and all clouds. Aggregate distributions separated by water and land are shown to the right of each panel with the global mean values indicated.

Figure 16: Seasonal variations of $\epsilon$ for high-, mid- and low-level clouds averaged over the whole globe and over three latitude zones (tropical $= \pm 15^{\circ}$, mid lat $= \pm 30-60^{\circ}$, high lat $= \pm 60-90^{\circ}$ )

Figure 17: Geographic distribution of annual mean values of the spatial standard deviation of cloud top temperatures (K) for high-, mid-, low-level clouds and all clouds. Aggregate distributions separated by water and land are shown to the right of each panel with the global 
mean values indicated.

Figure 18: Zonal, daily mean, upwelling shortwave flux at the top-of-atmosphere (top panel) and its change for overcast conditions produced by cloud spatial variations at scales $<300 \mathrm{~km}$ (lower panel), both in $\mathrm{Wm}^{-2}$, calculated using the ISCCP data for 15 July 1986

Figure 19: Zonal, daily mean, upwelling longwave flux at the top-of-atmosphere (top panel) and its change for overcast conditions produced by cloud spatial variations at scales $<300 \mathrm{~km}$ (lower panel), both in $\mathrm{Wm}^{-2}$, calculated using the ISCCP data for 15 July 1986.

Figure A1: Cloud albedo error as function of the spatial inhomogeneity parameter, $\epsilon$, for a planeparallel cloud (upper left panel), for a cloud with varying optical thicknesses in a gammadistribution using logarithmic mean and variance (upper right panel), for a gamma-distribution using linear mean and variance (lower left panel) and for a gamma-distribution using linear and logarithmic mean values (lower right panel). 

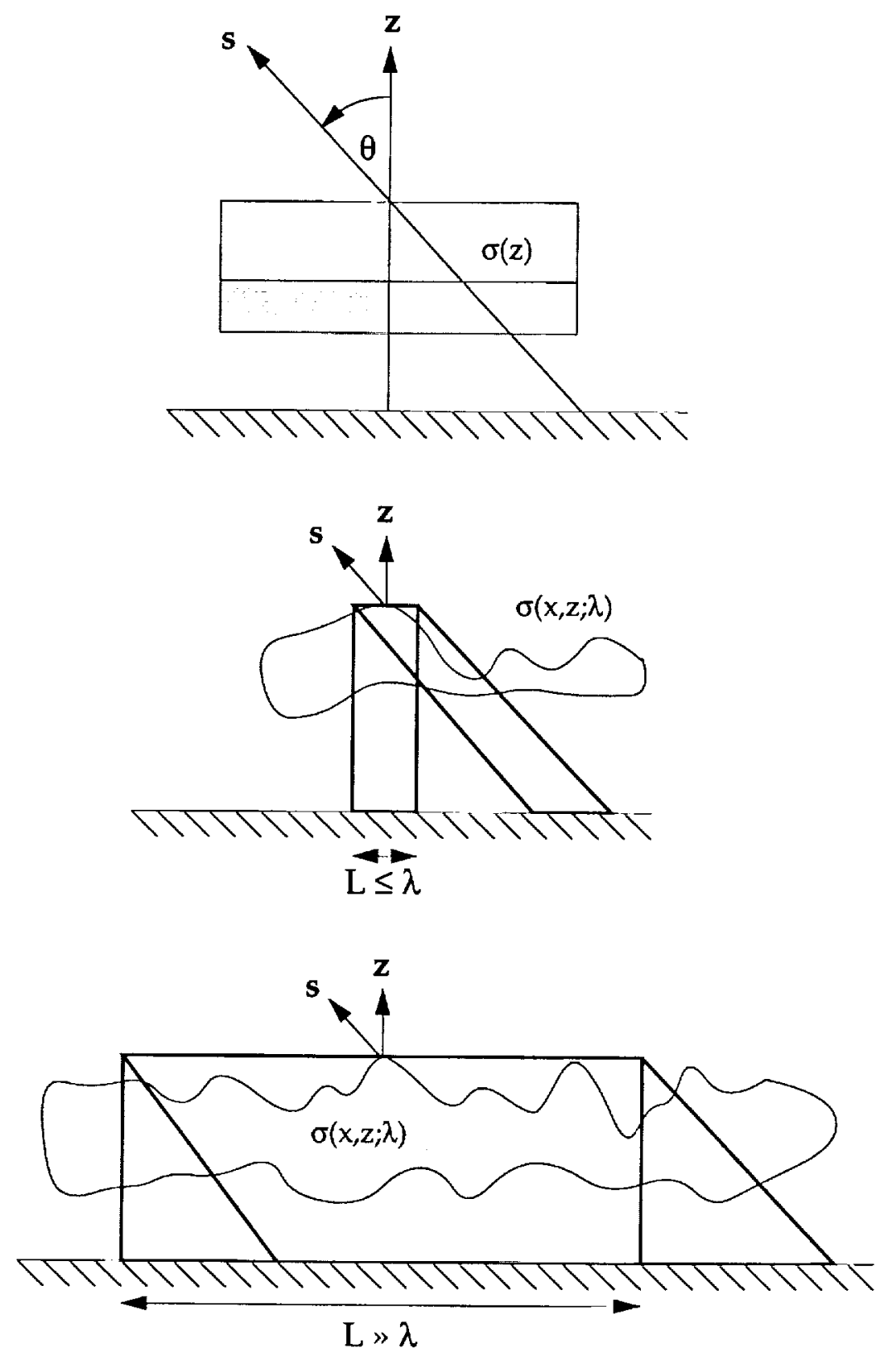

Fig I 


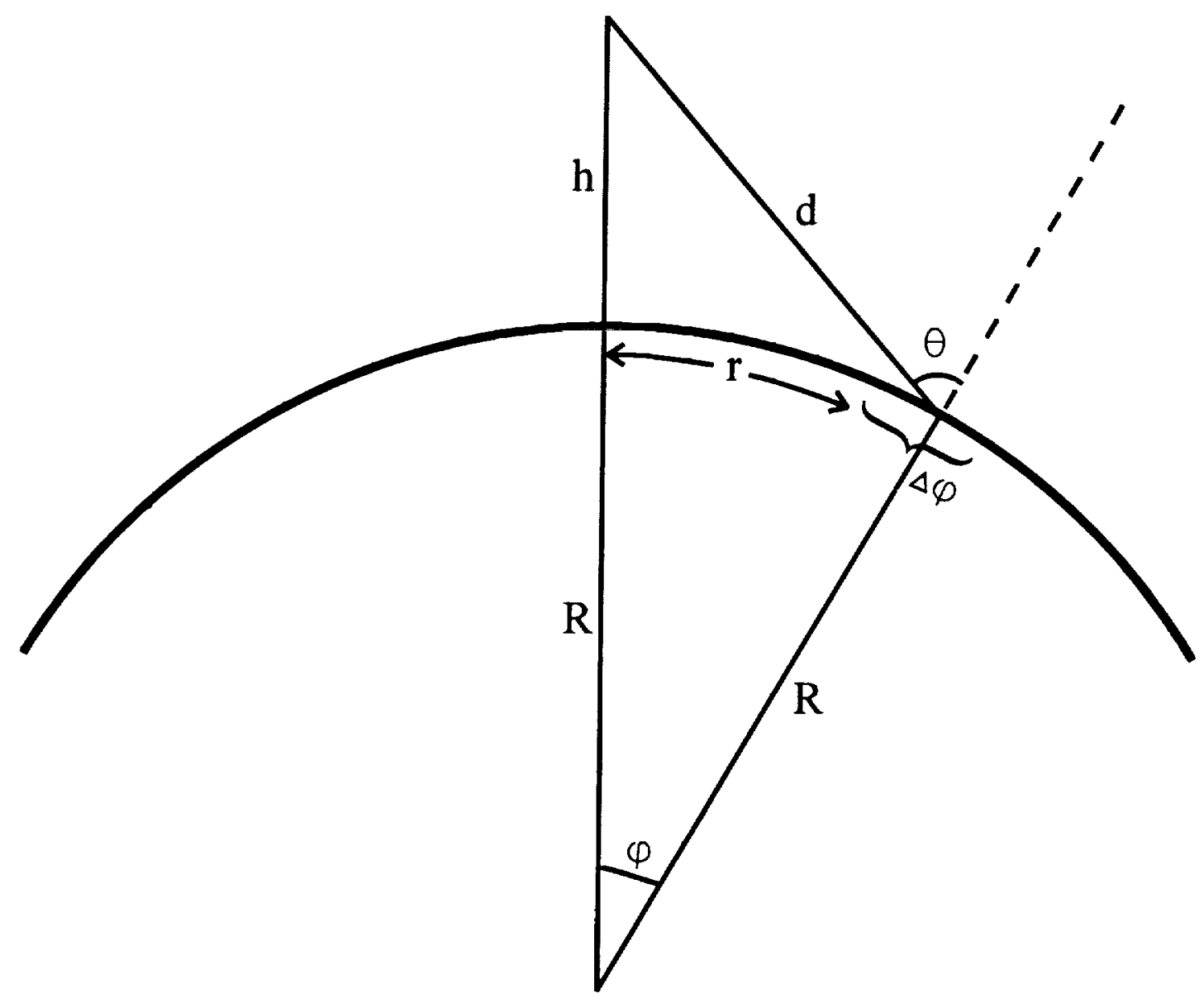

FIG 2 


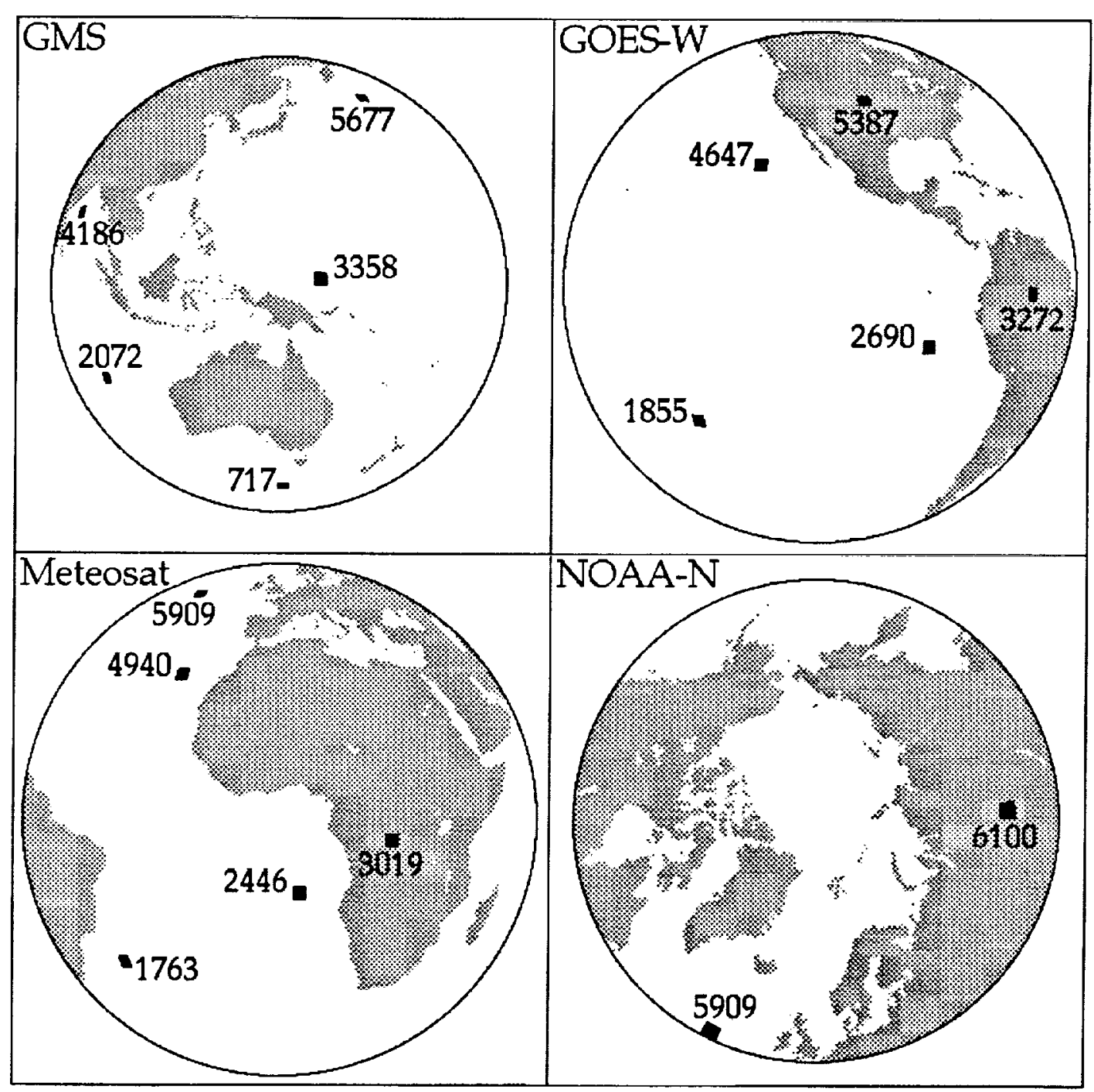

Fig 3 


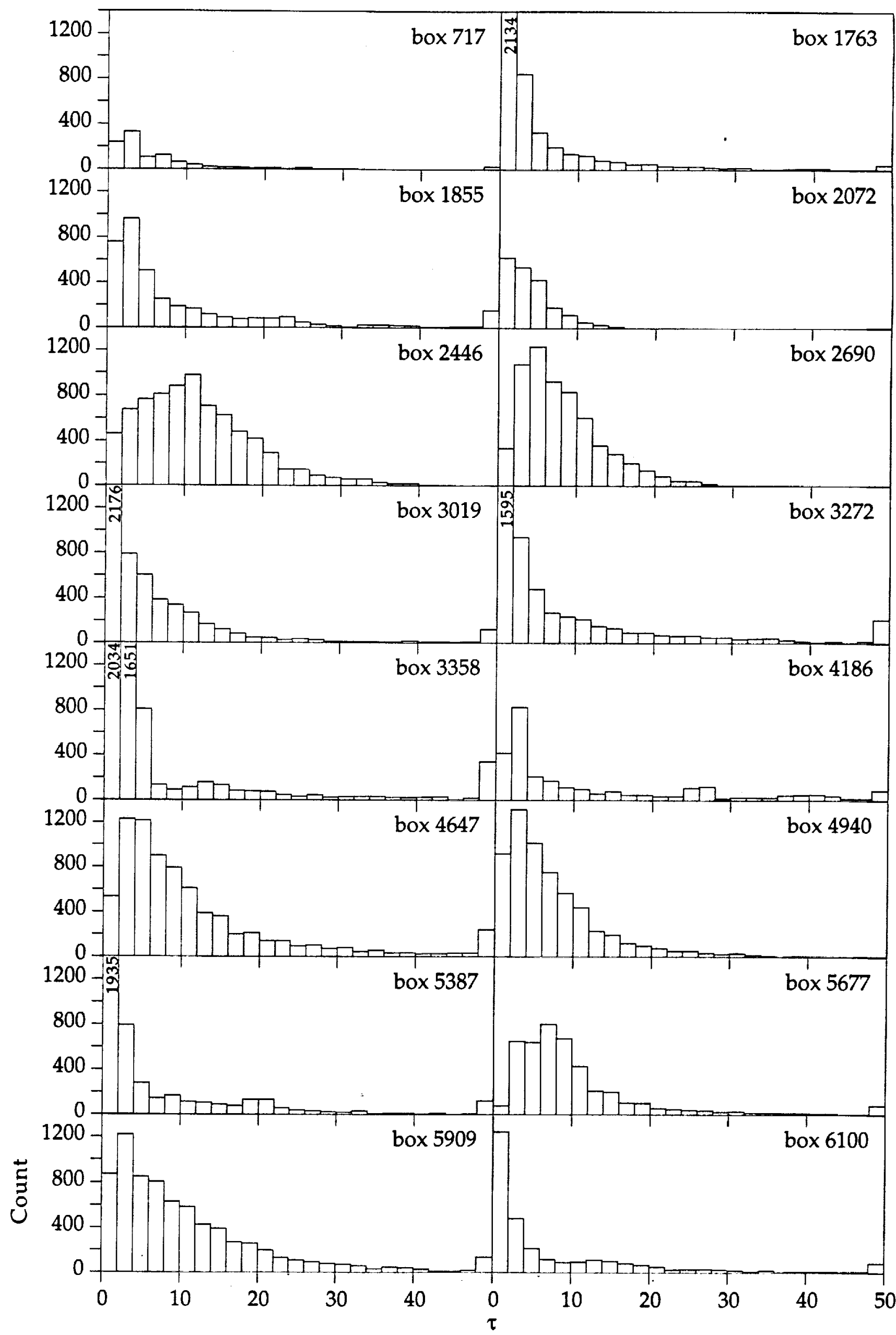

FI 64 

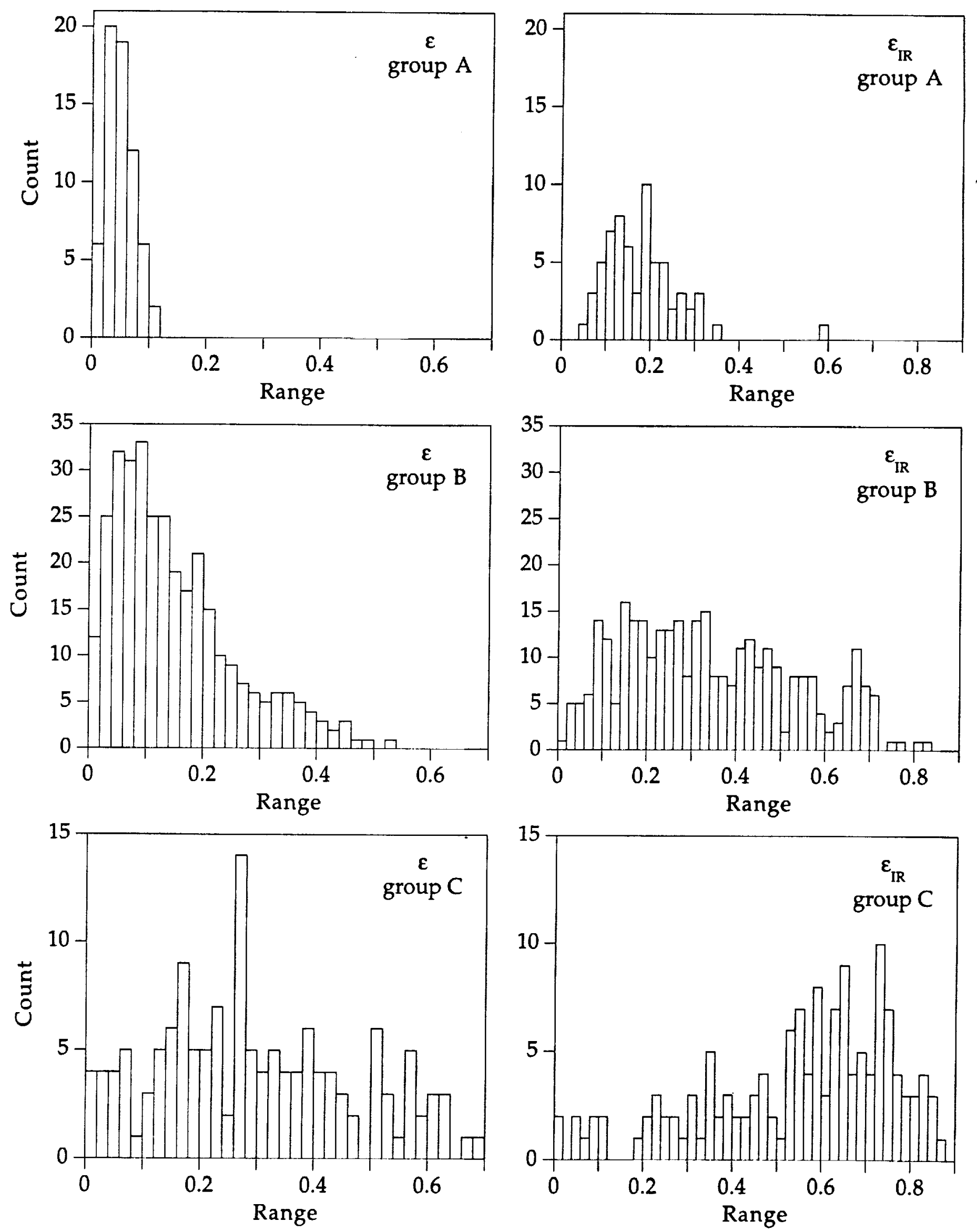

FIG 5 . 

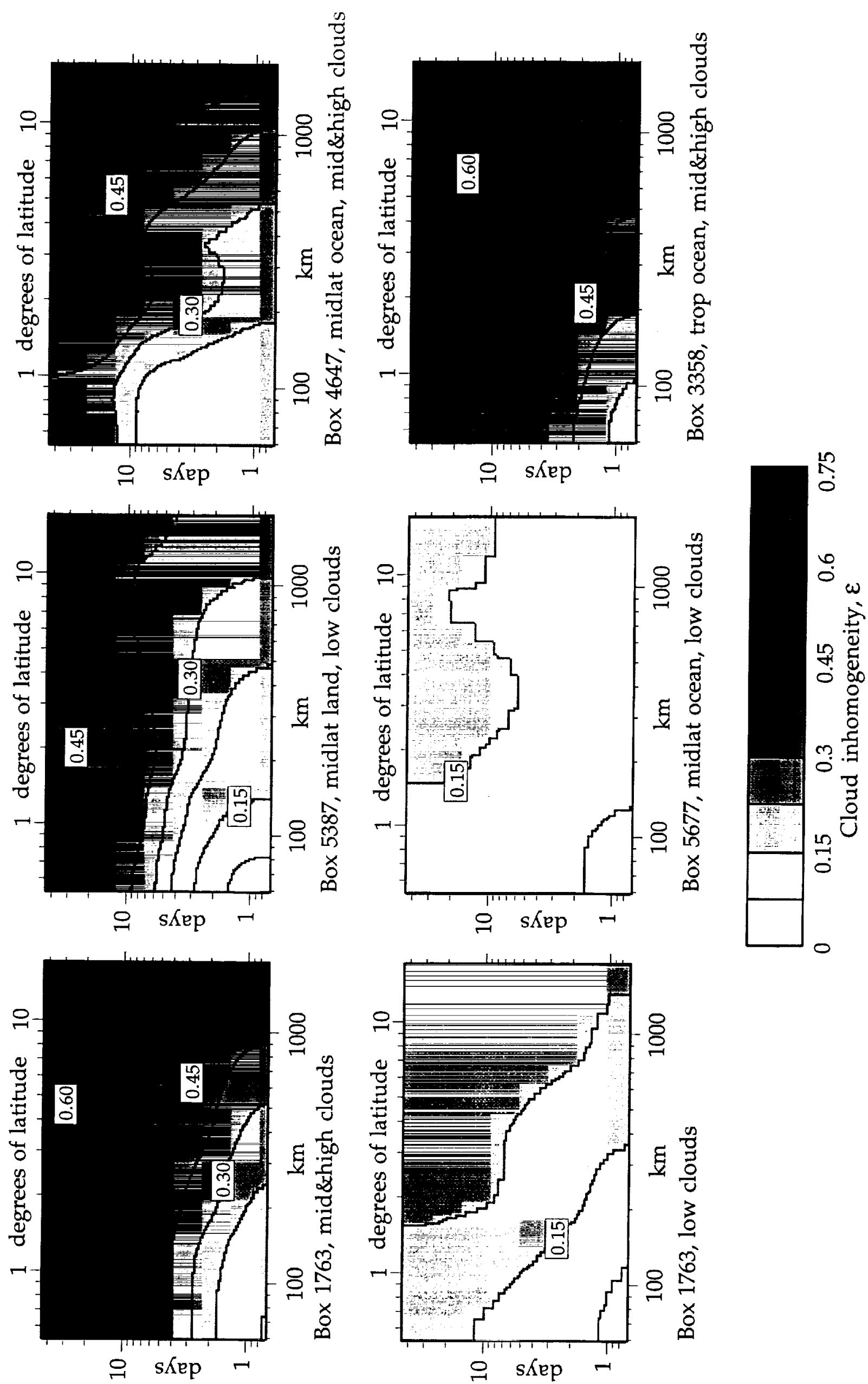

FiE 6 

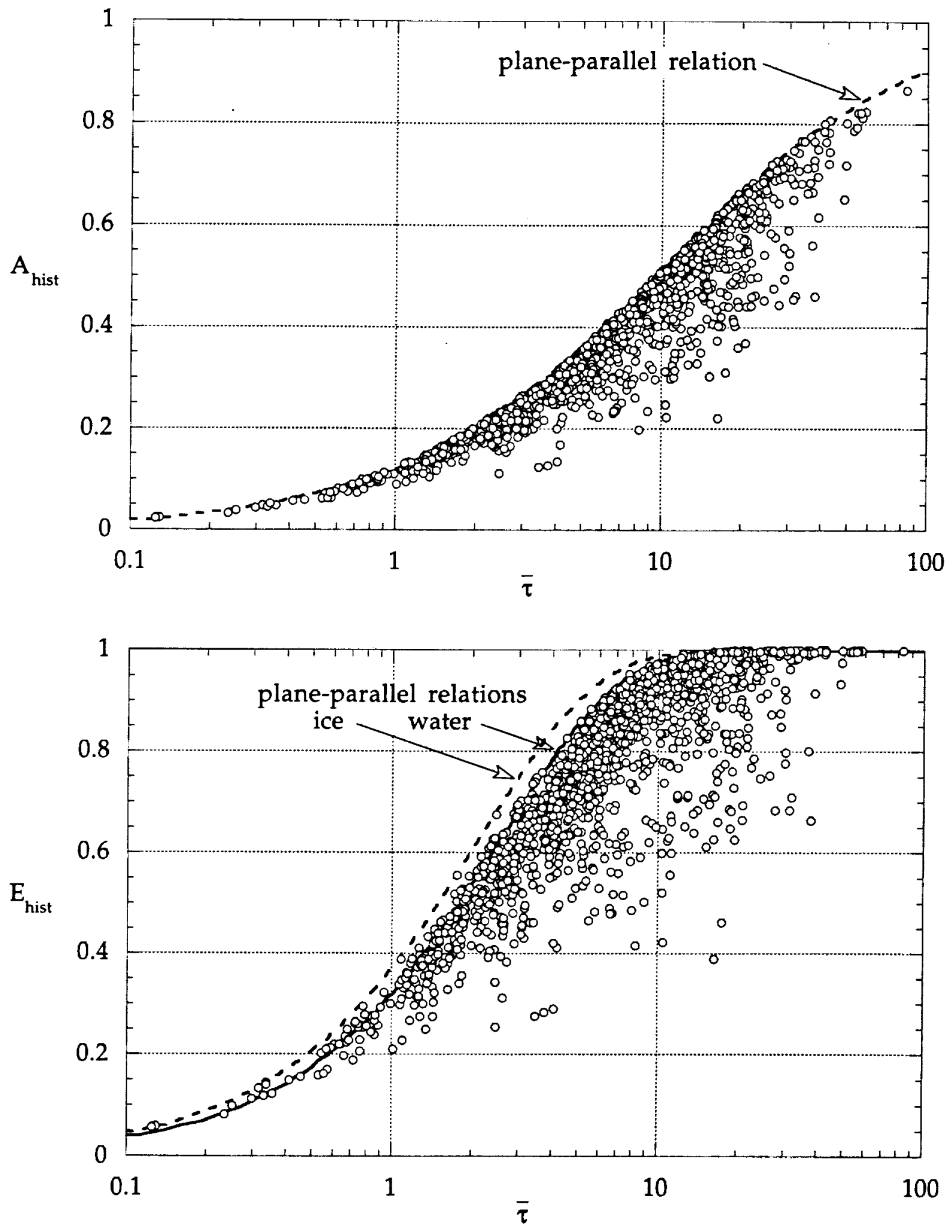

FIE 7 

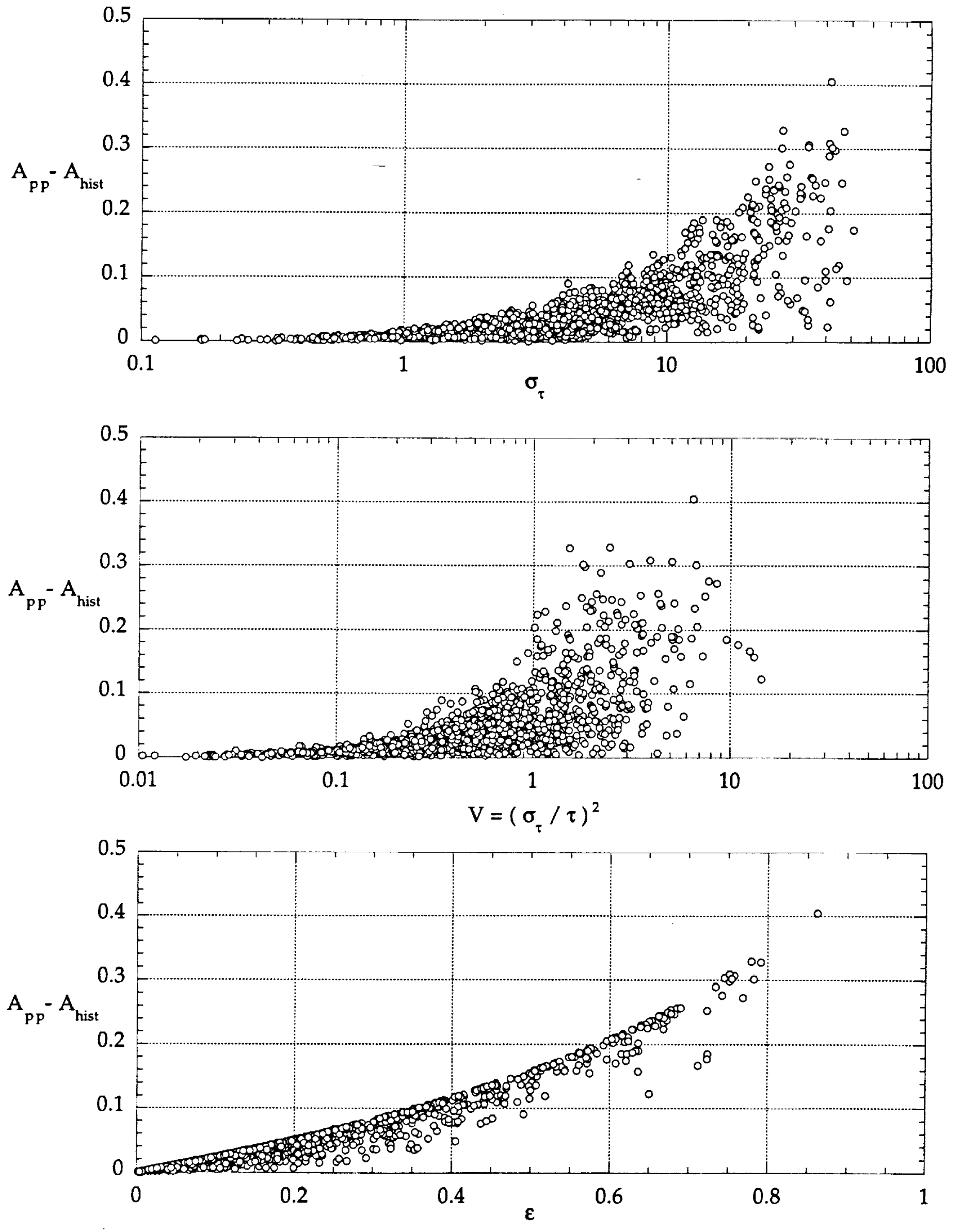

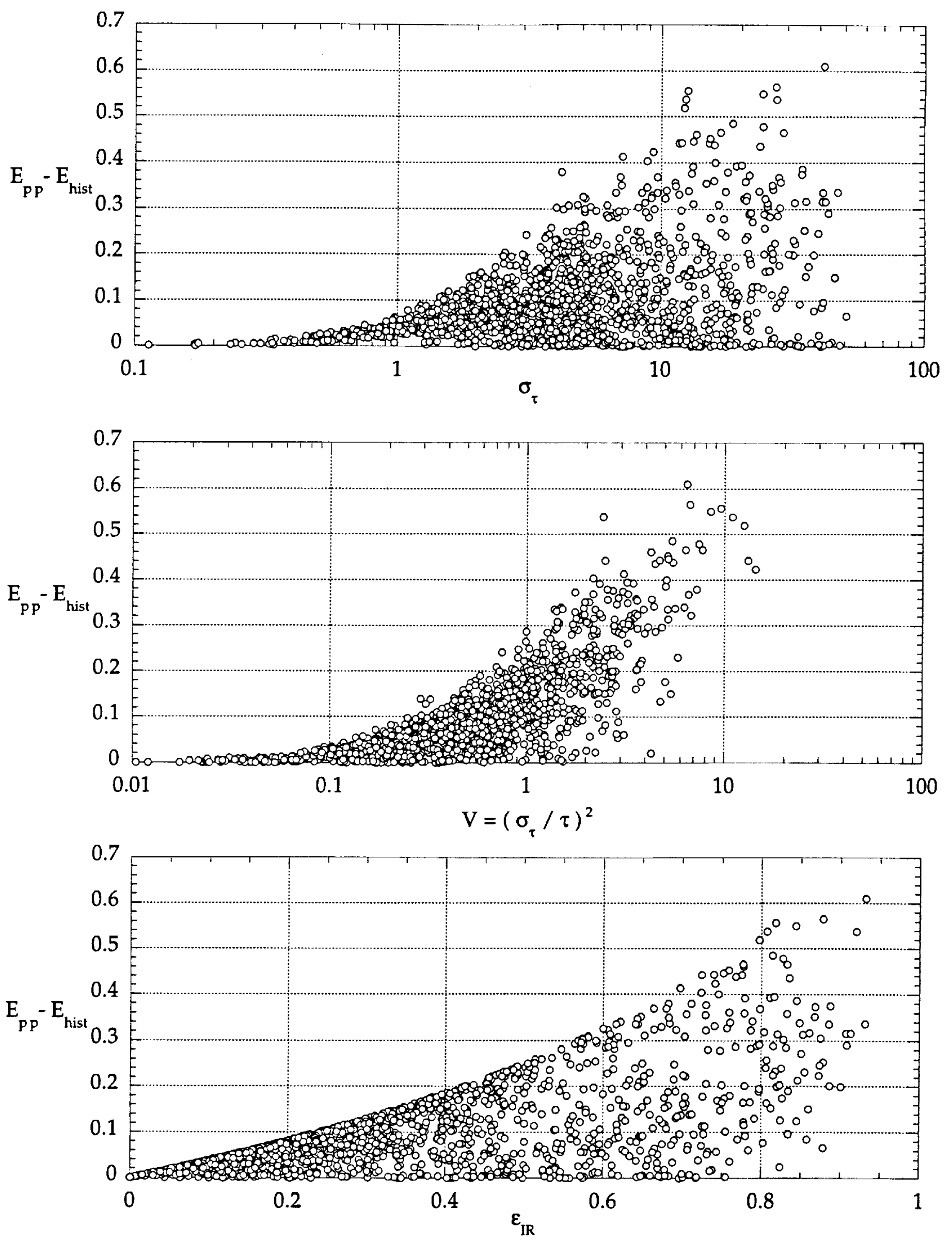

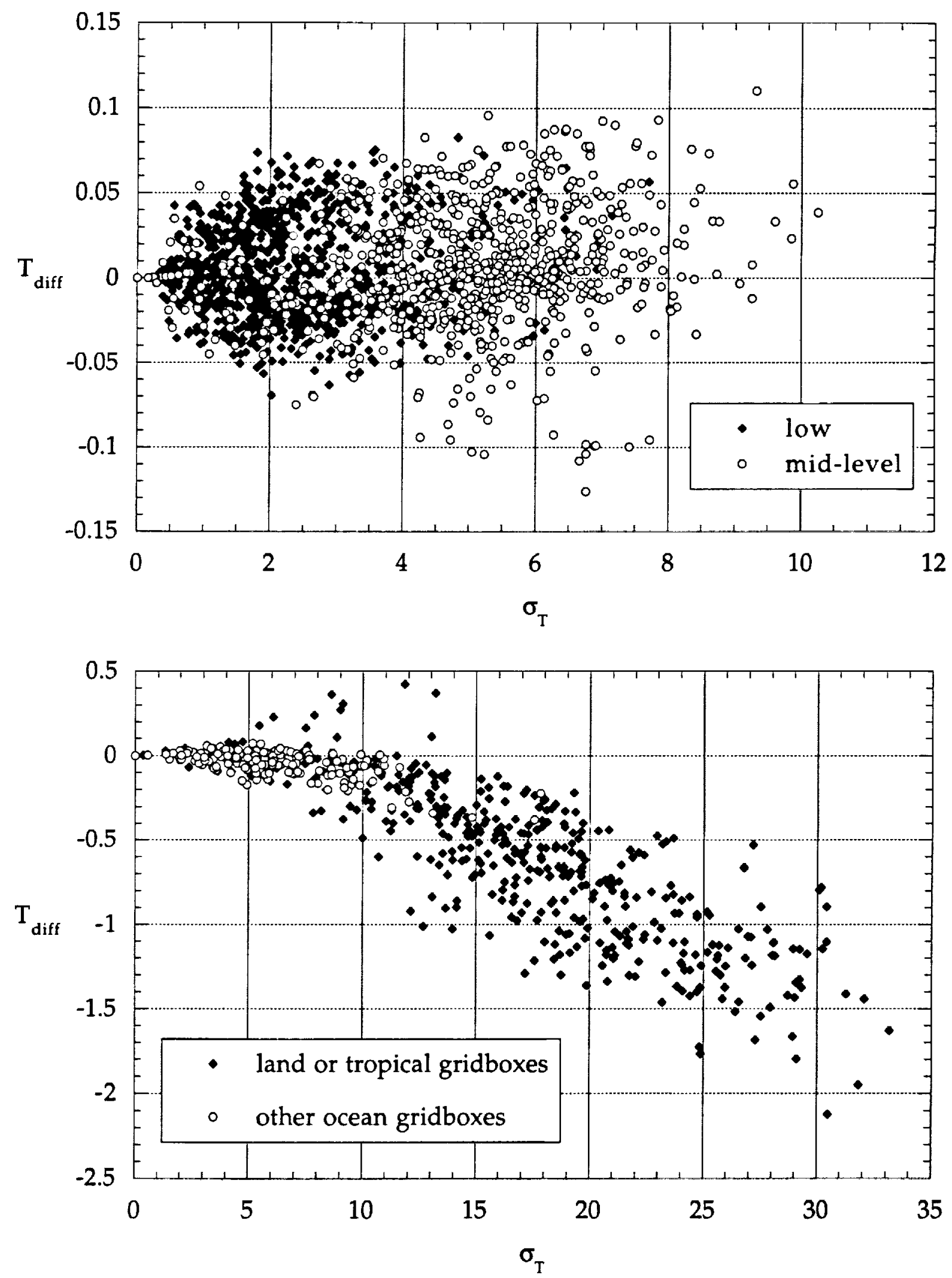

$F \mid \in 10$ 


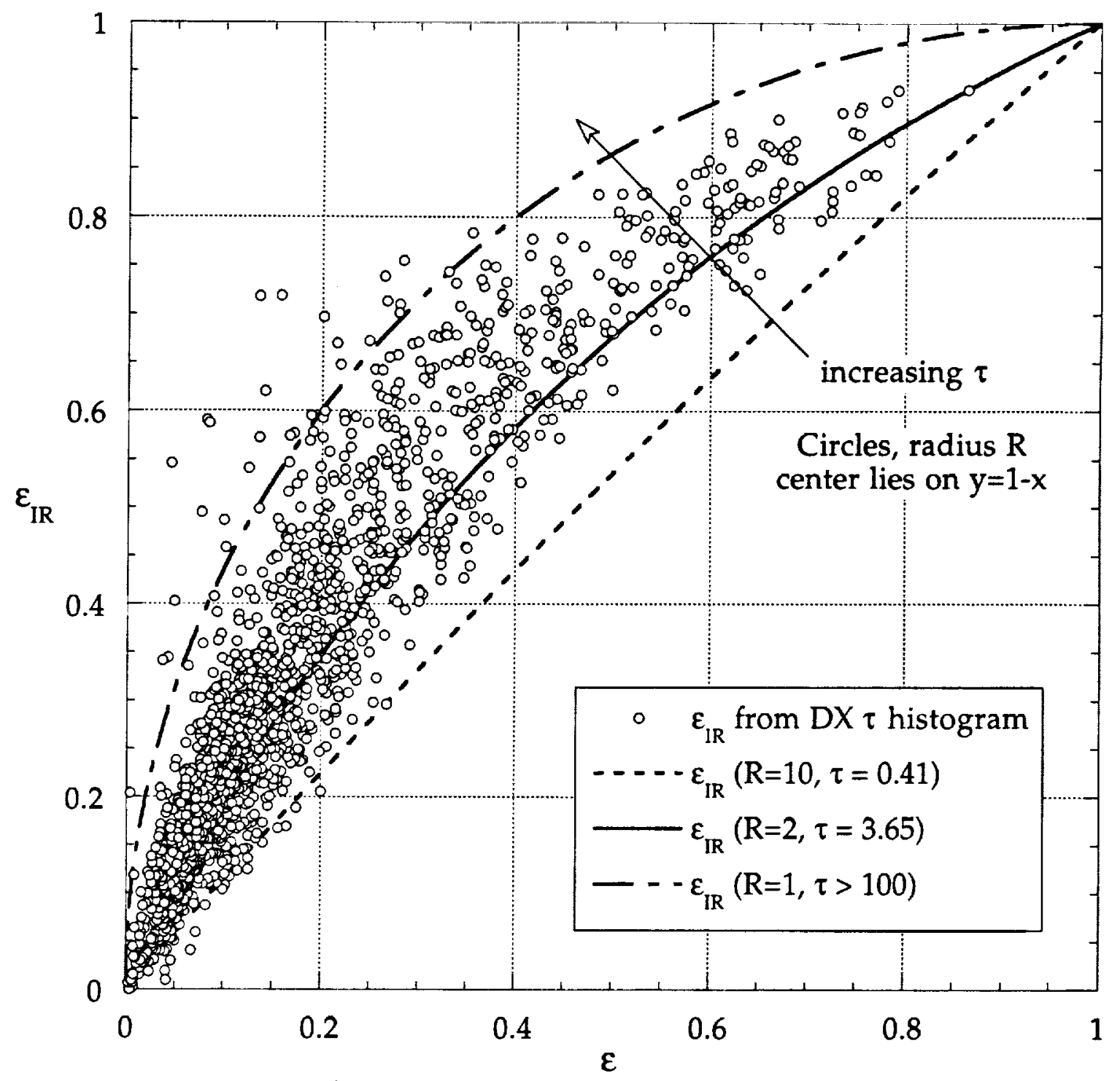

$F / G \|$ 


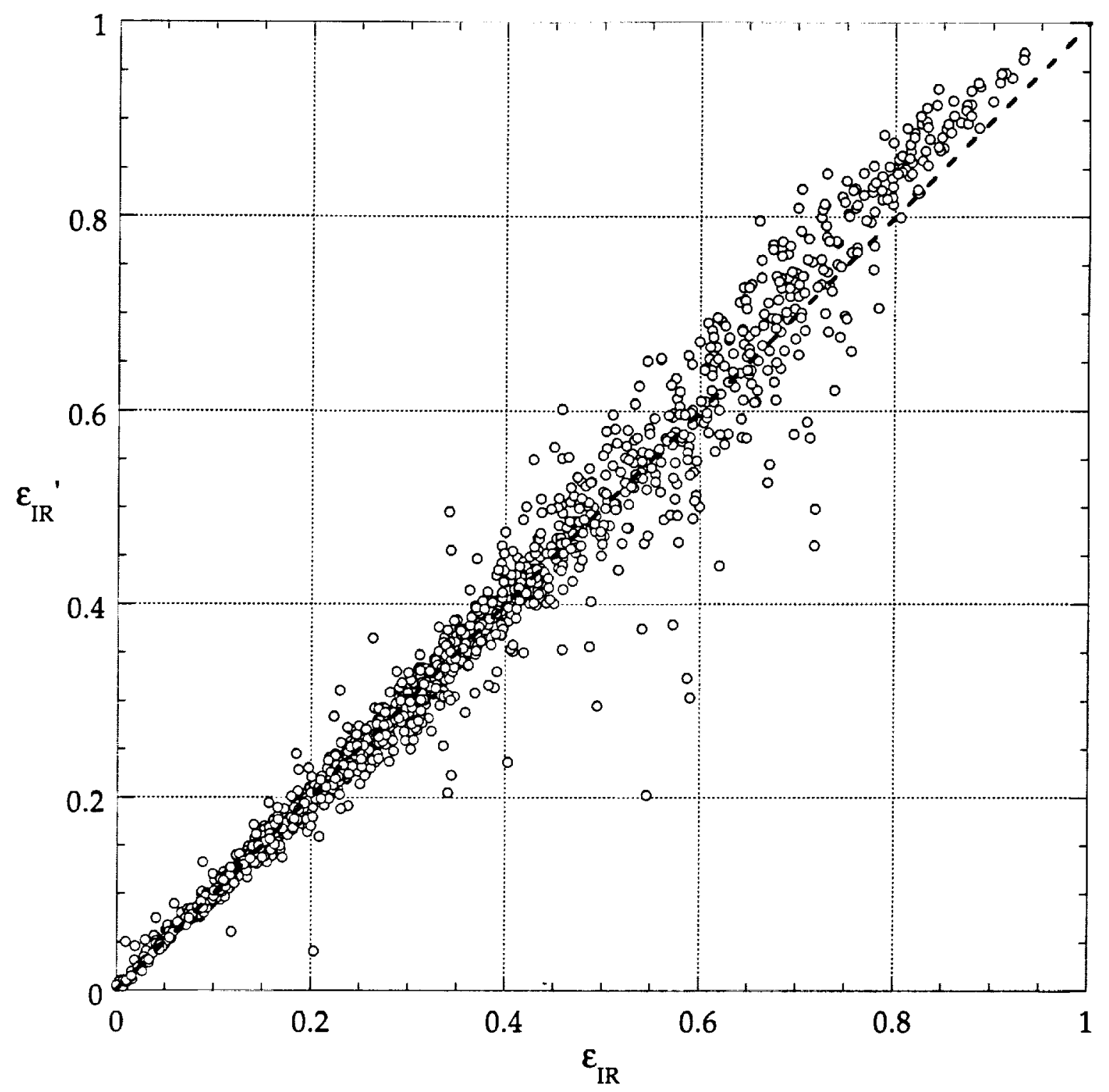

$F / G \quad 12 A$ 


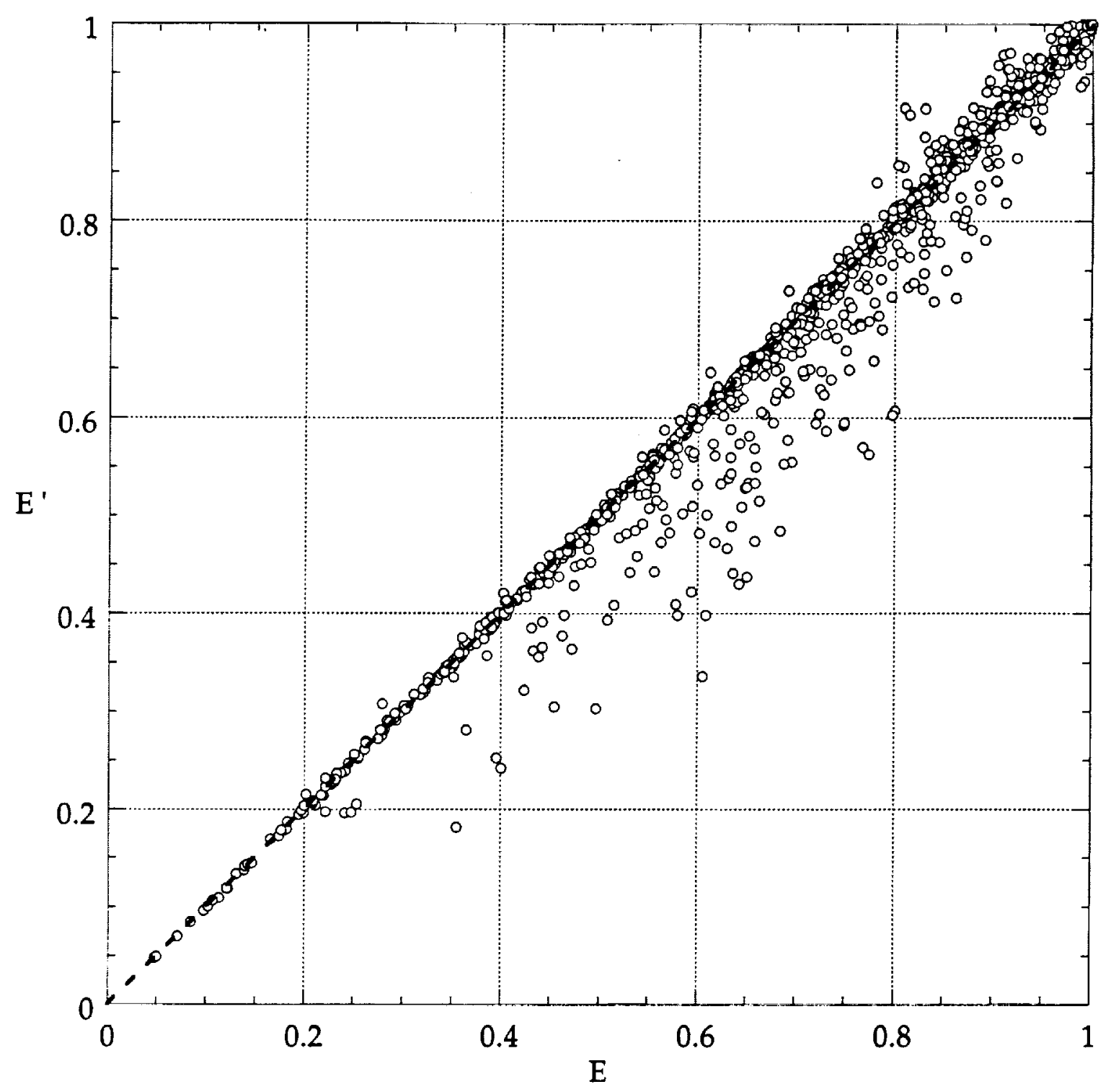

FIG $12 B$ 

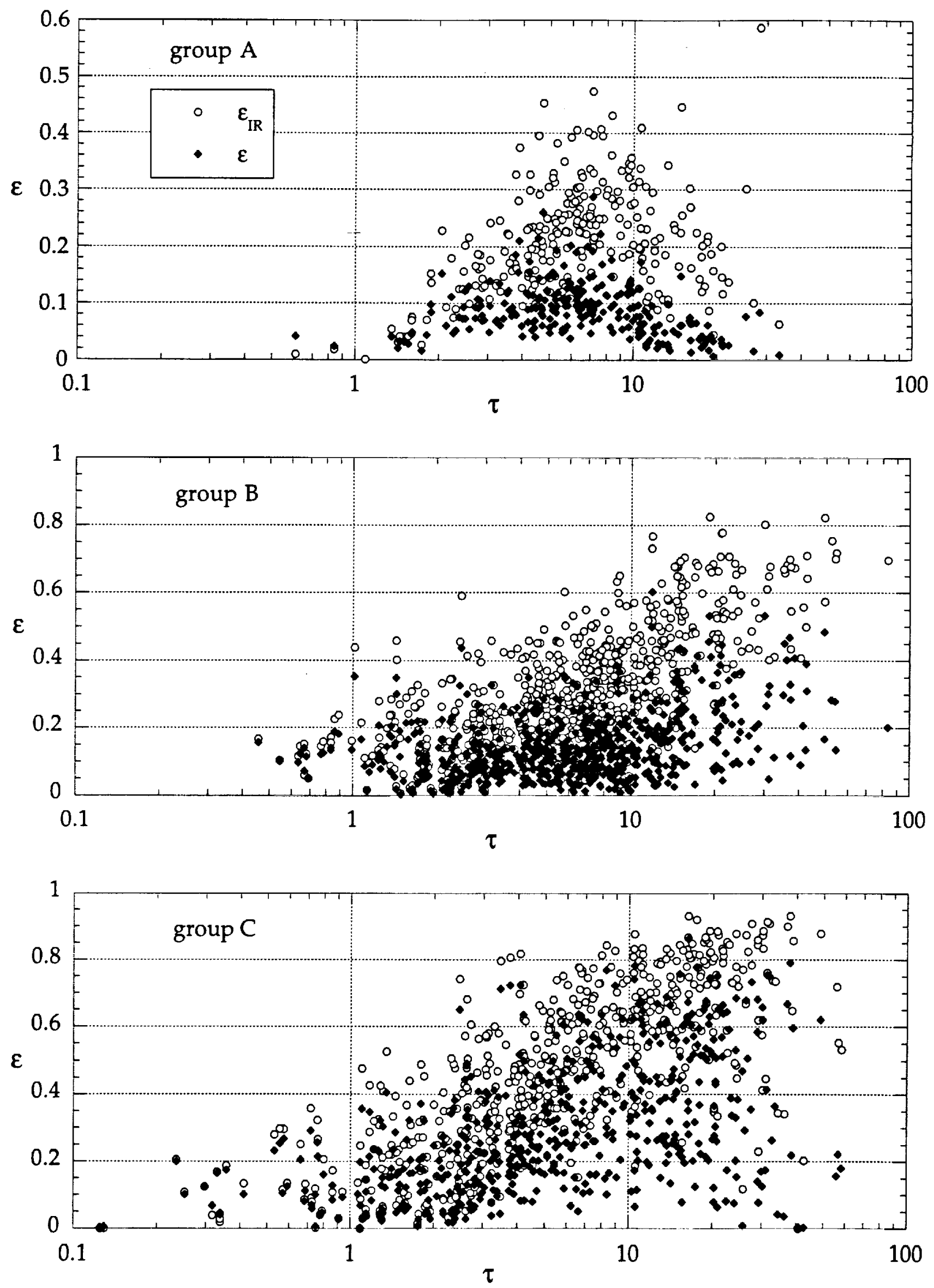

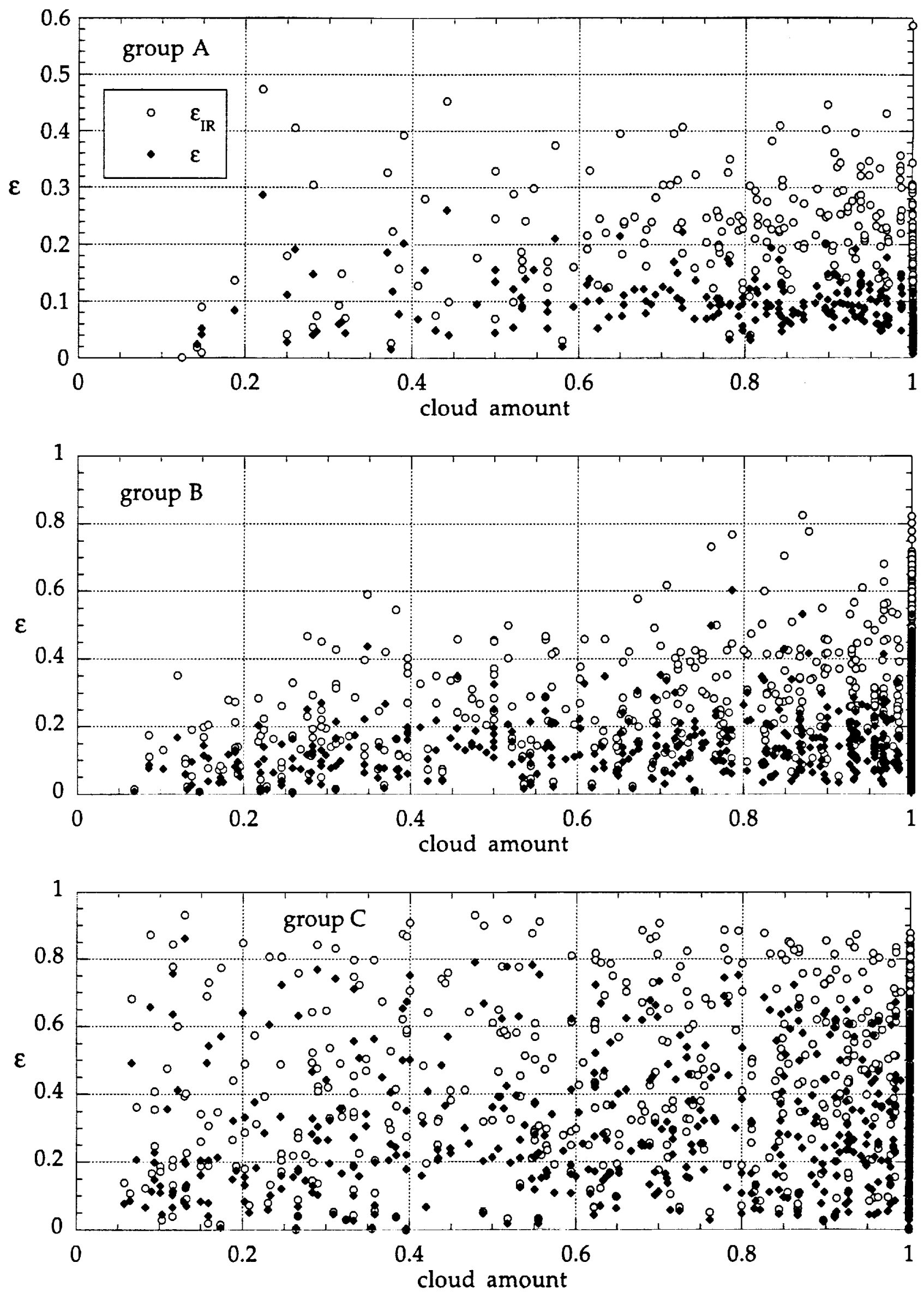


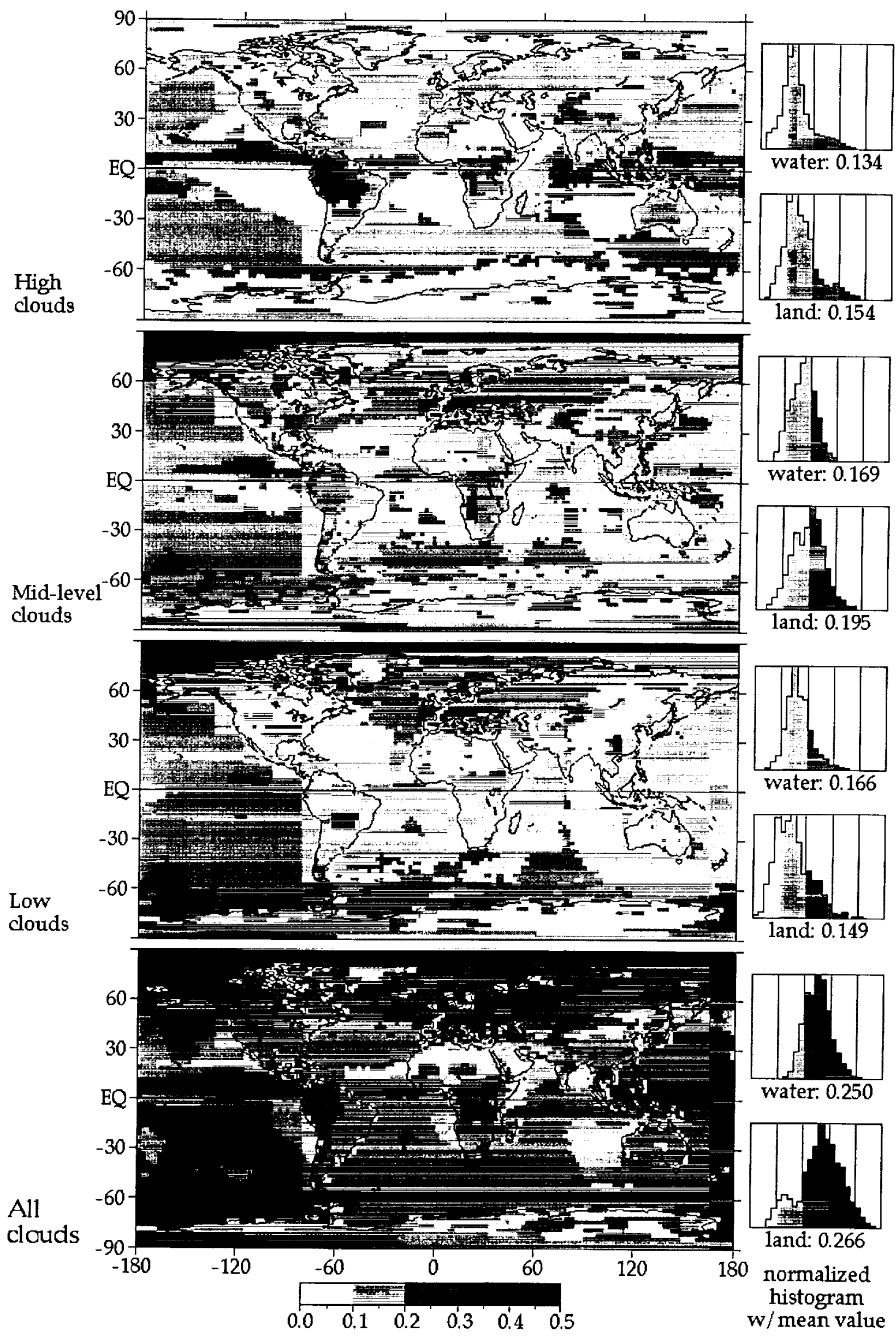


a) high clouds over water

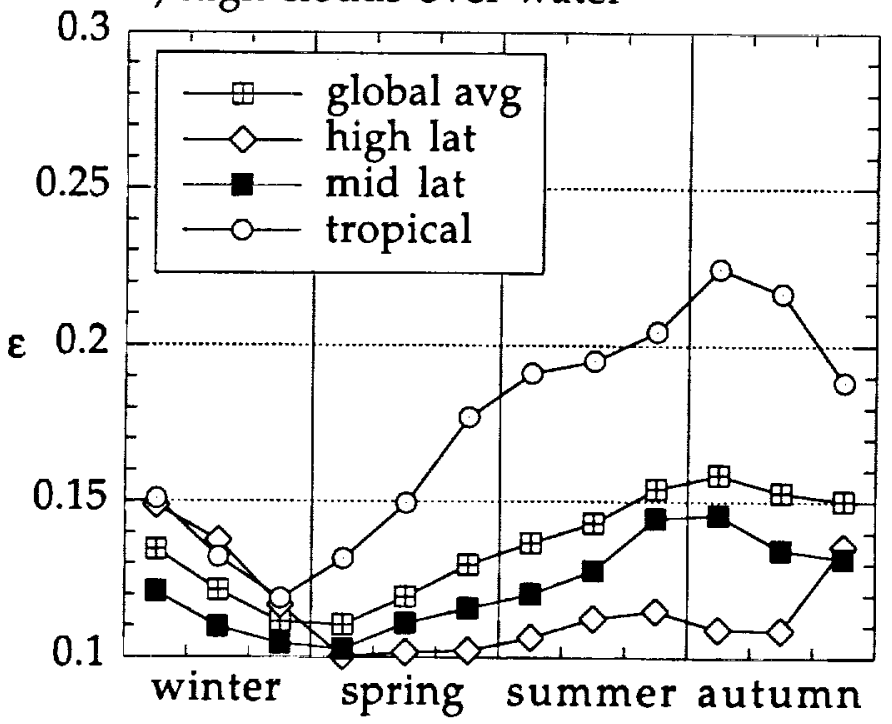

c) mid-levels clouds over water

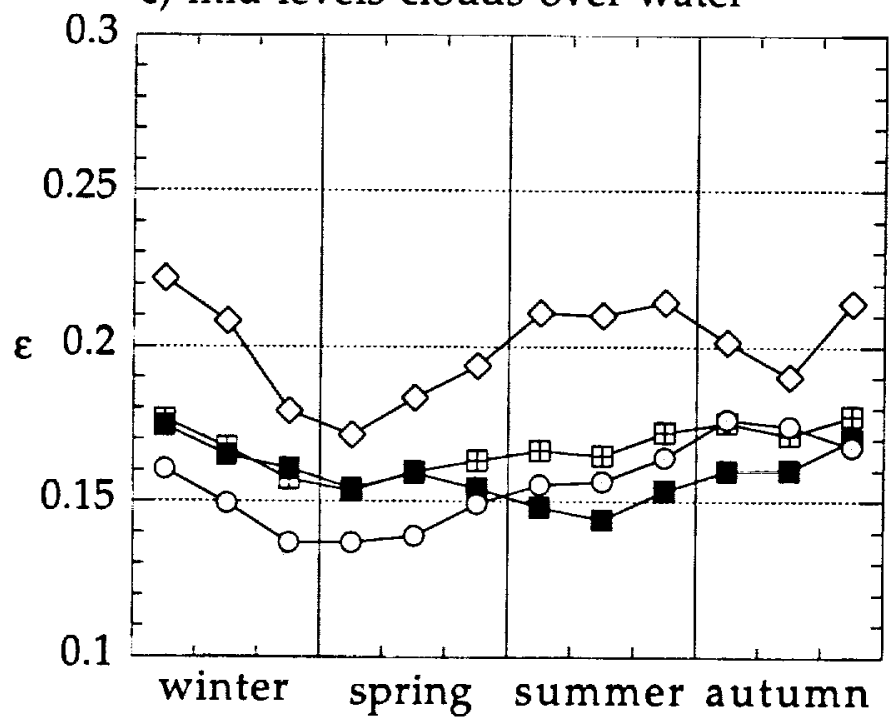

e) low clouds over water

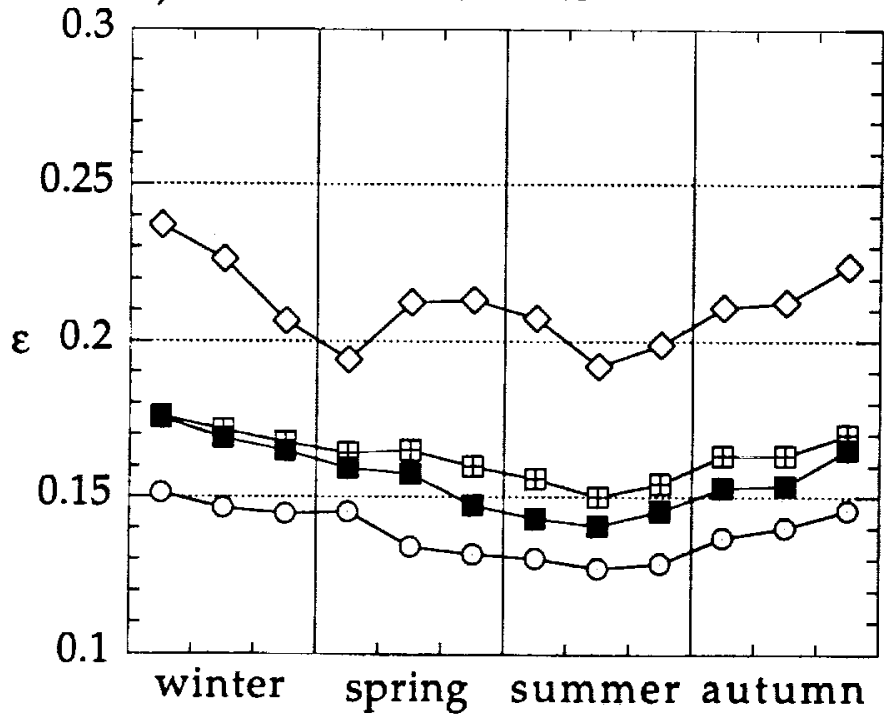

b) high clouds over land

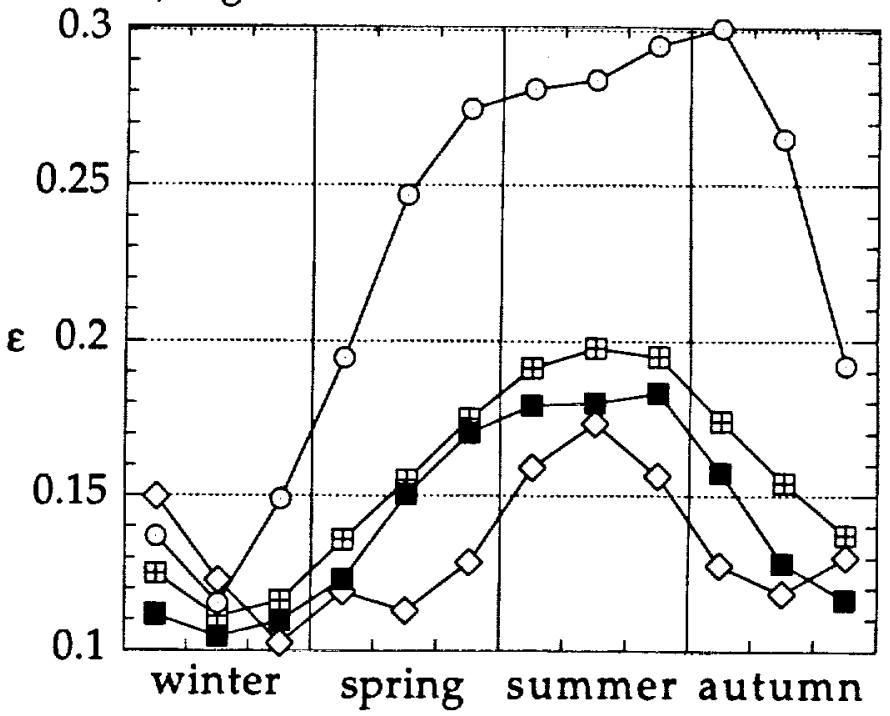

d) mid-level clouds over land

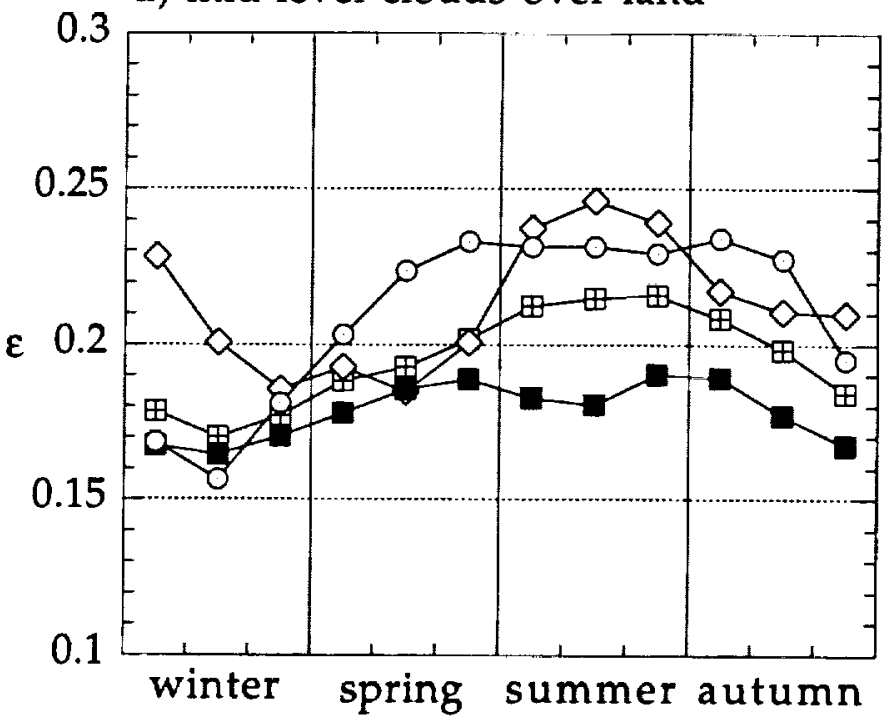

f) low clouds over land

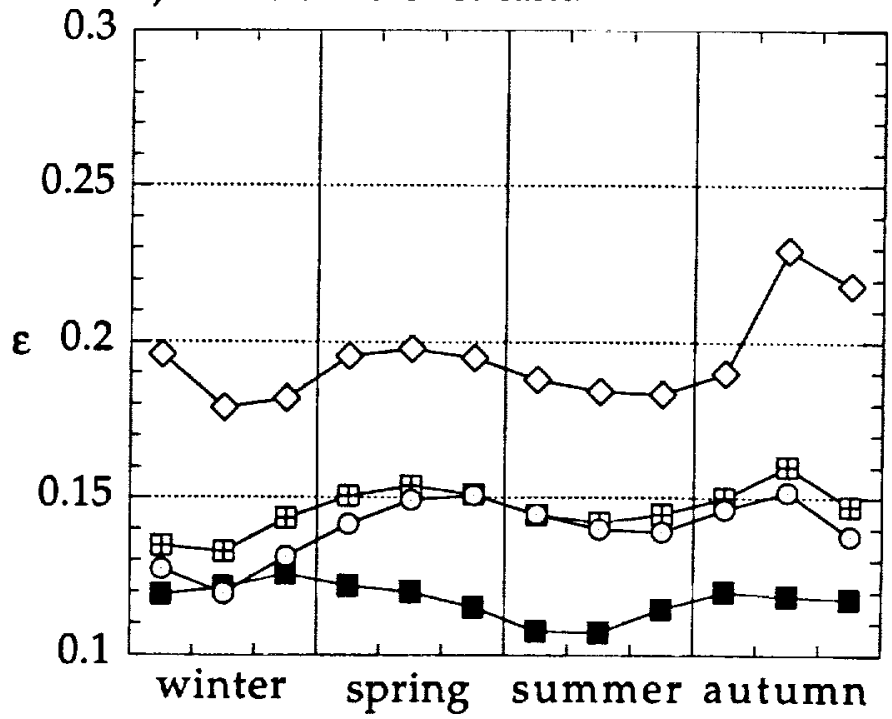



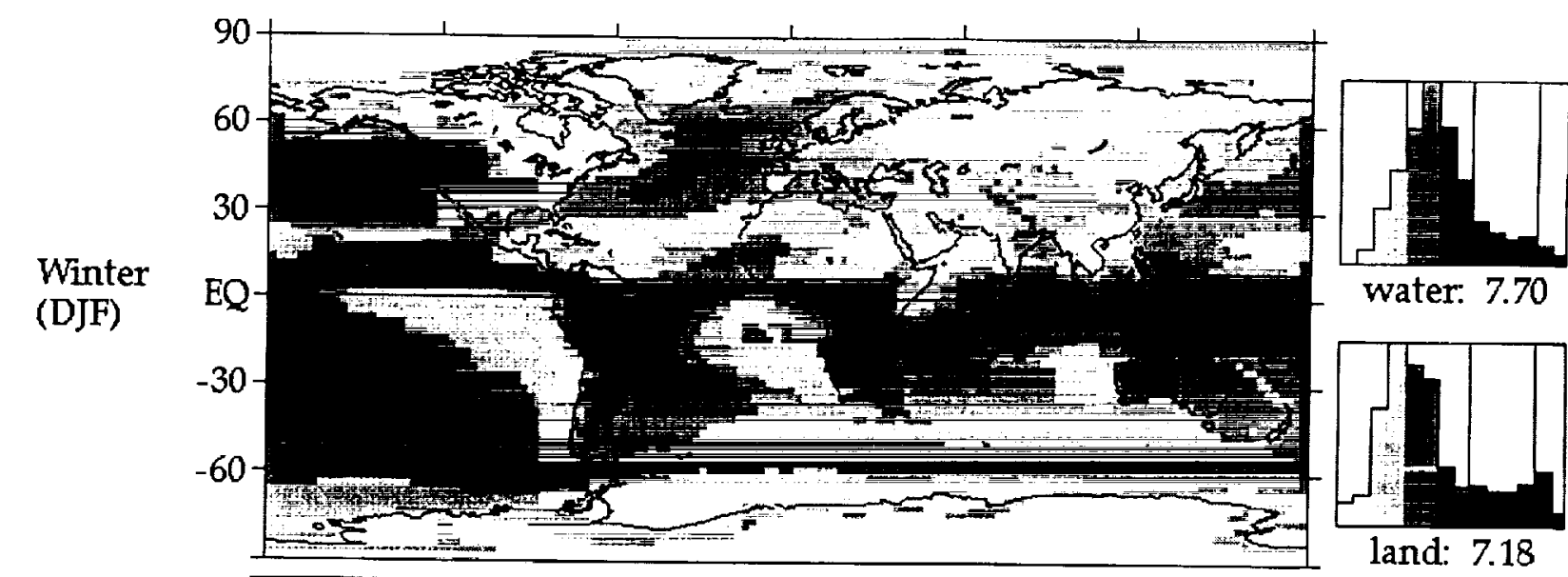

water: 7.70
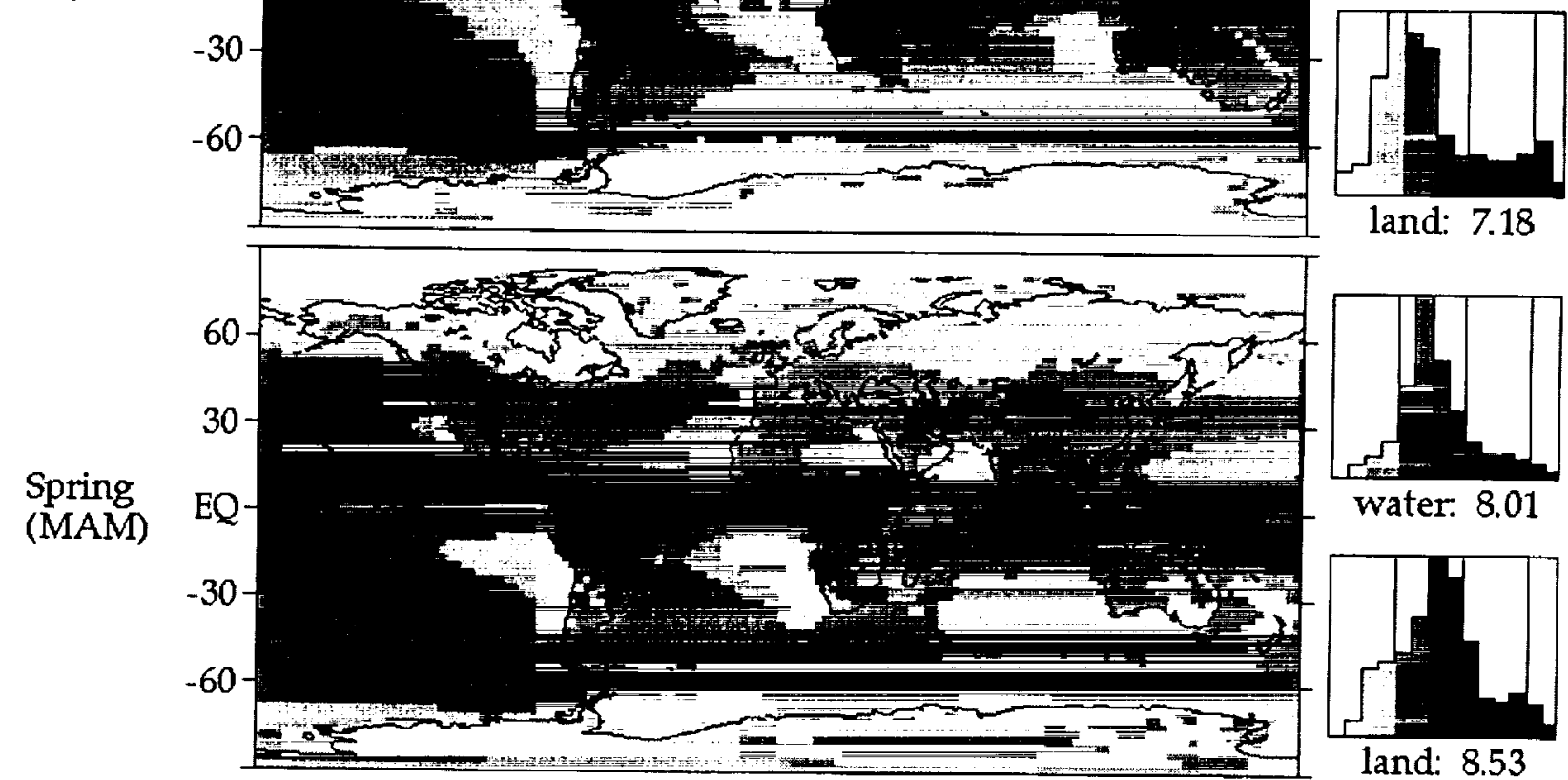

land: 7.18
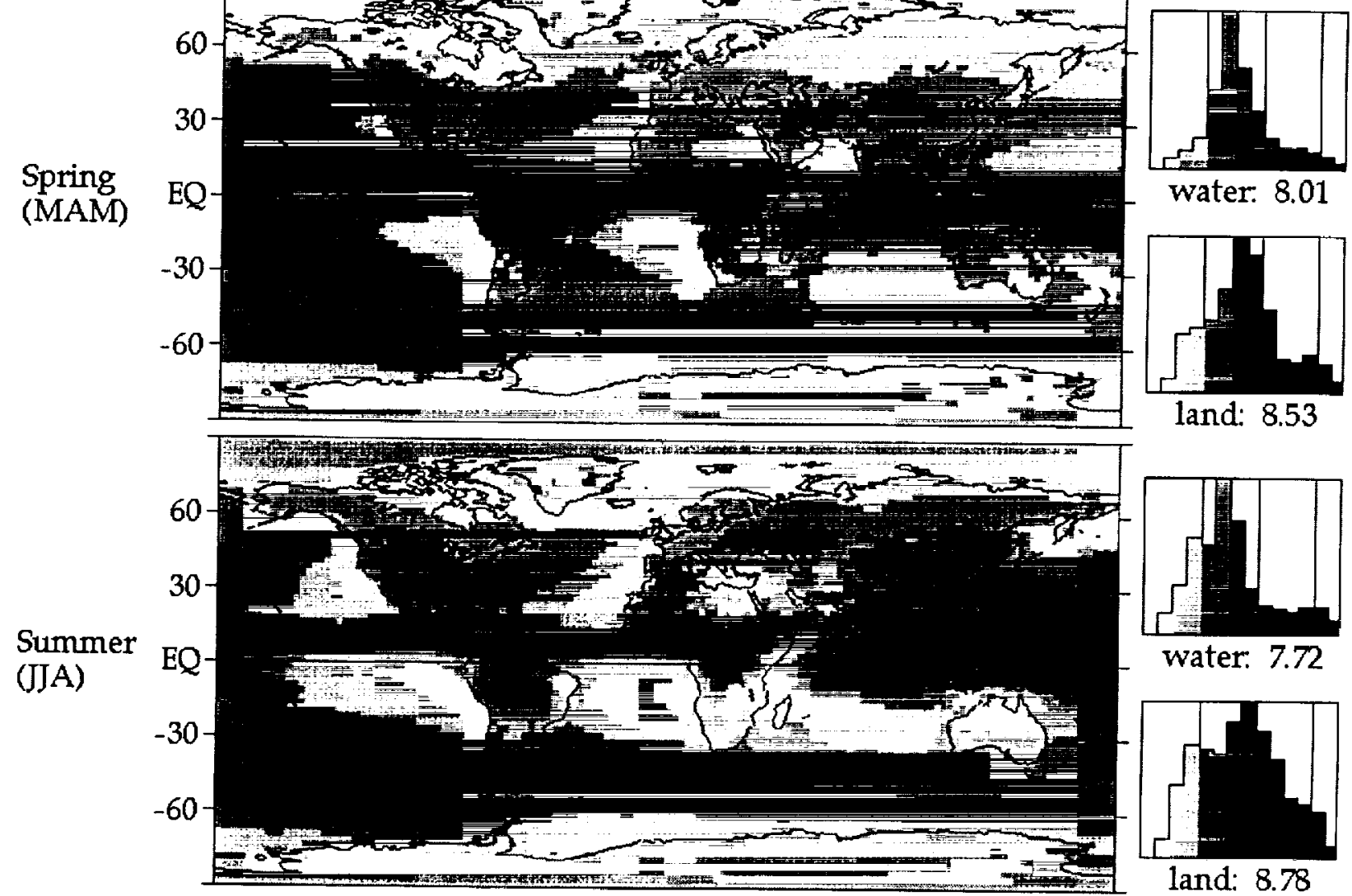

water: 8.01
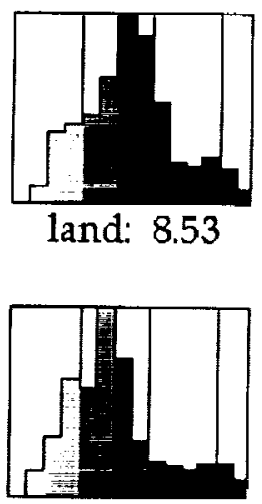

water: 7.72

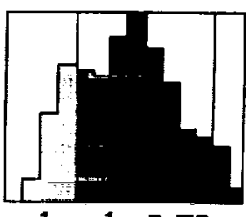

land: 8.78

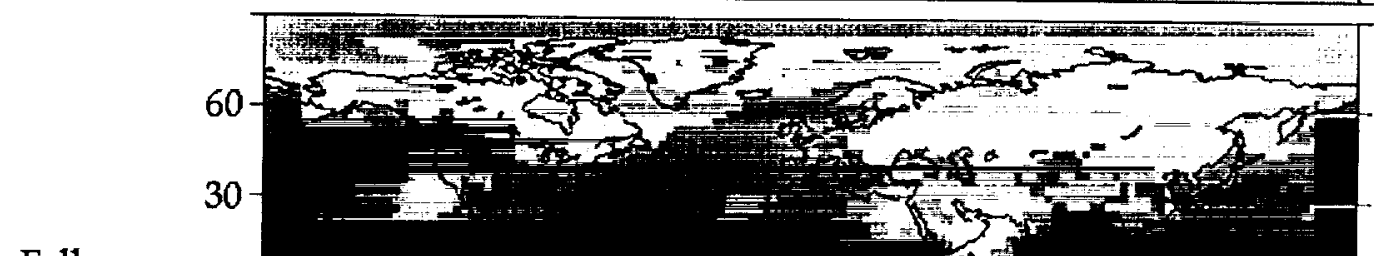

Fall (SON)
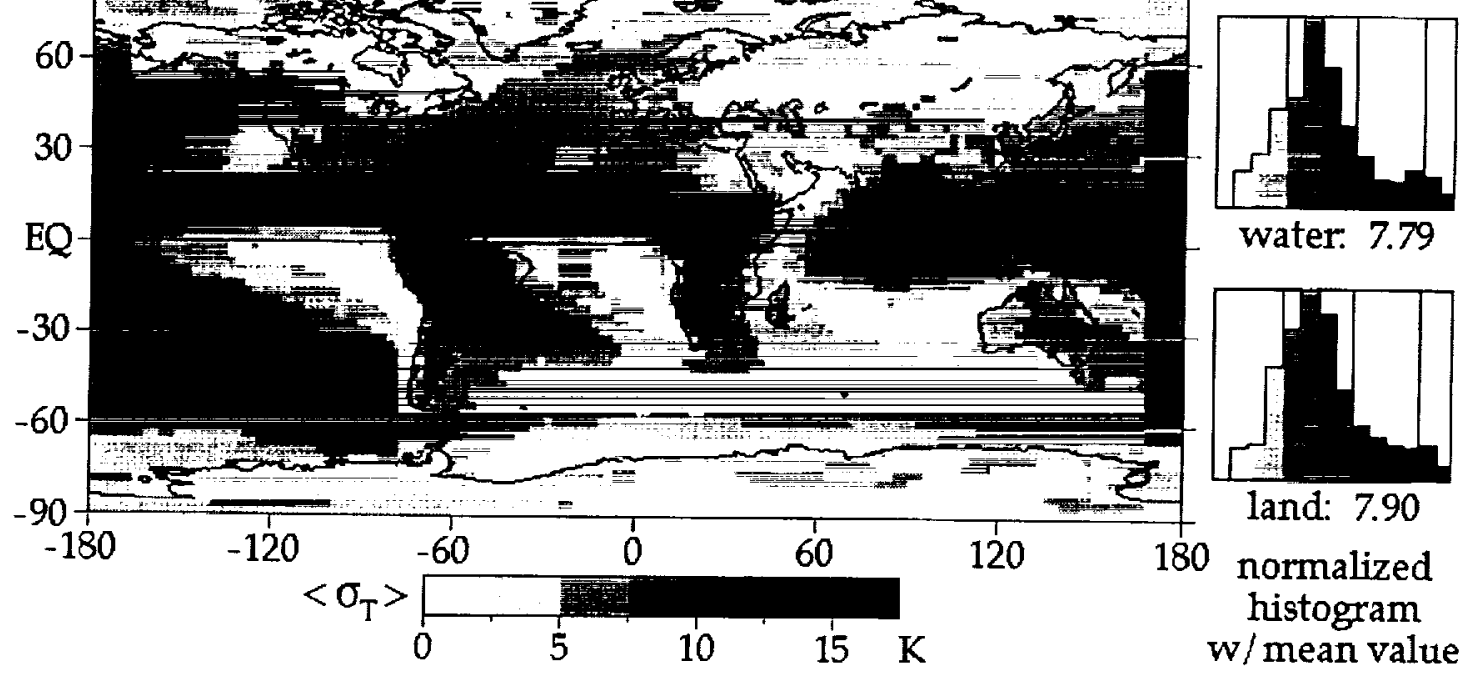

water: 7.79

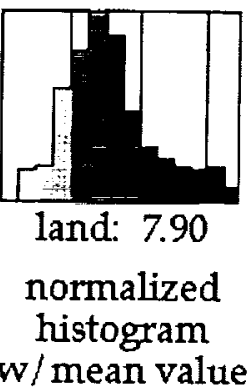


SHORTWAVE REFLECTED FLUXES AT TOP OF ATMOSPHERE -- OVERCAST
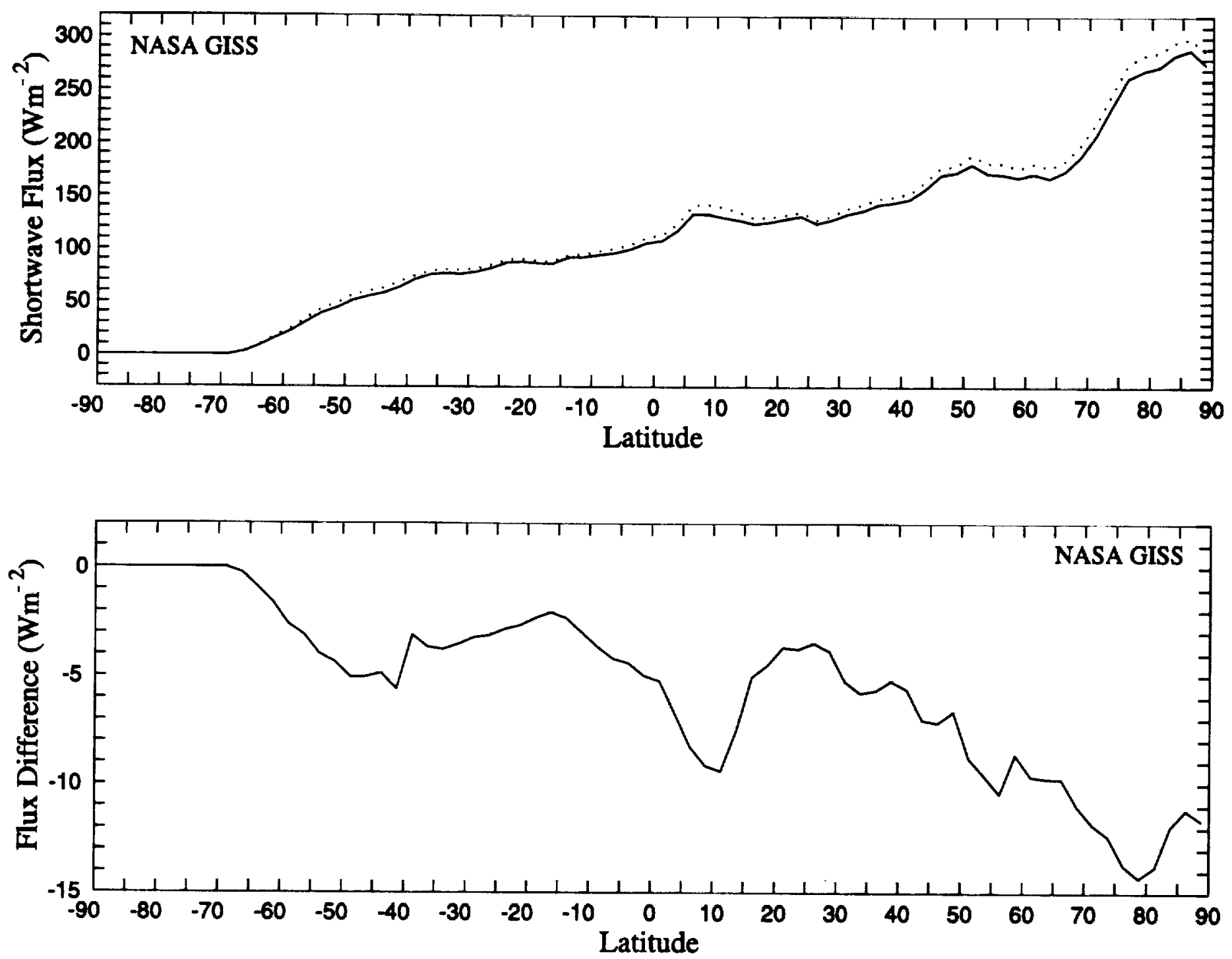

FiG 18 


\section{LONGWAVE EMITTED FLUXES AT TOP OF ATMOSPHERE -- OVERCAST}
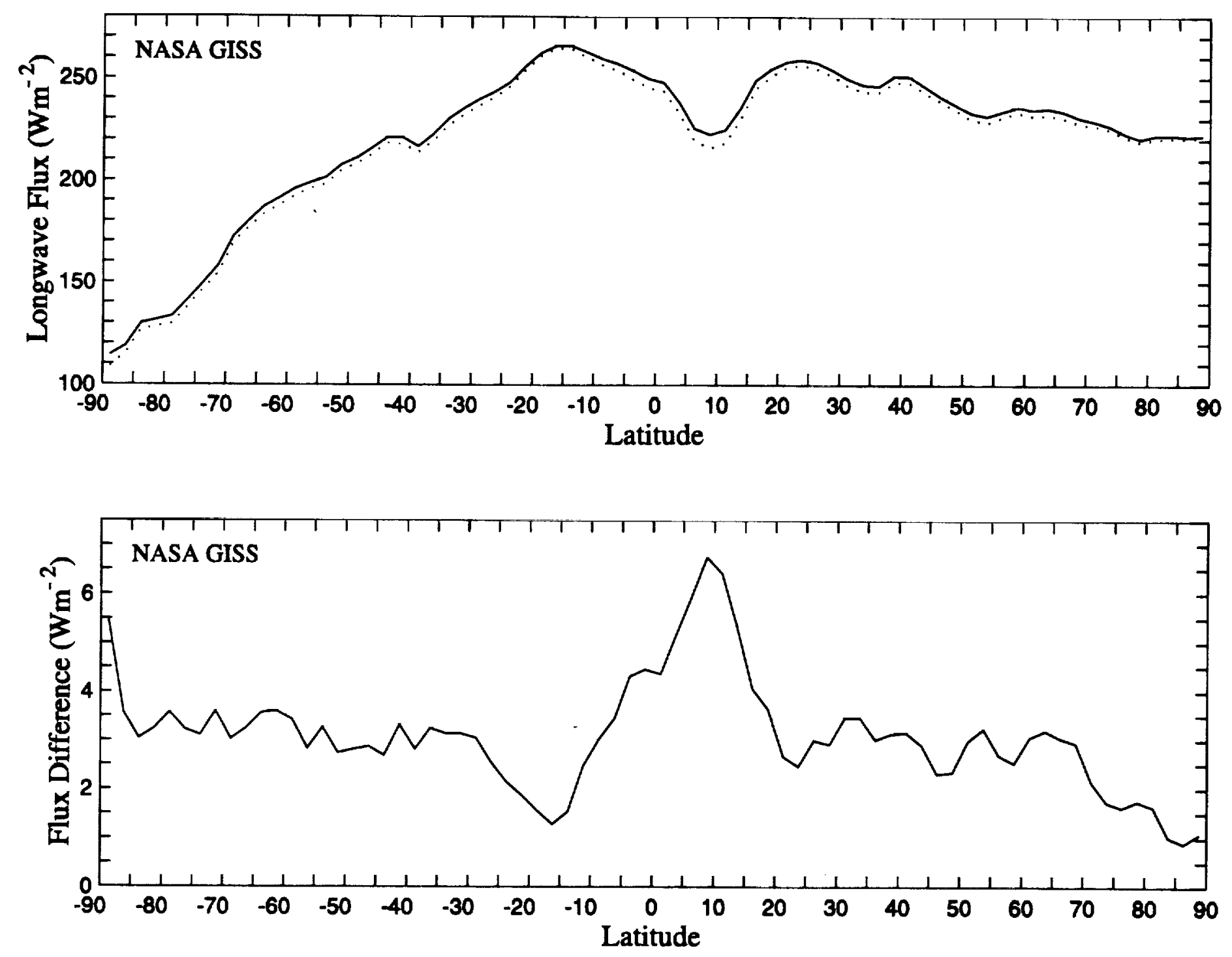

FiG 19 

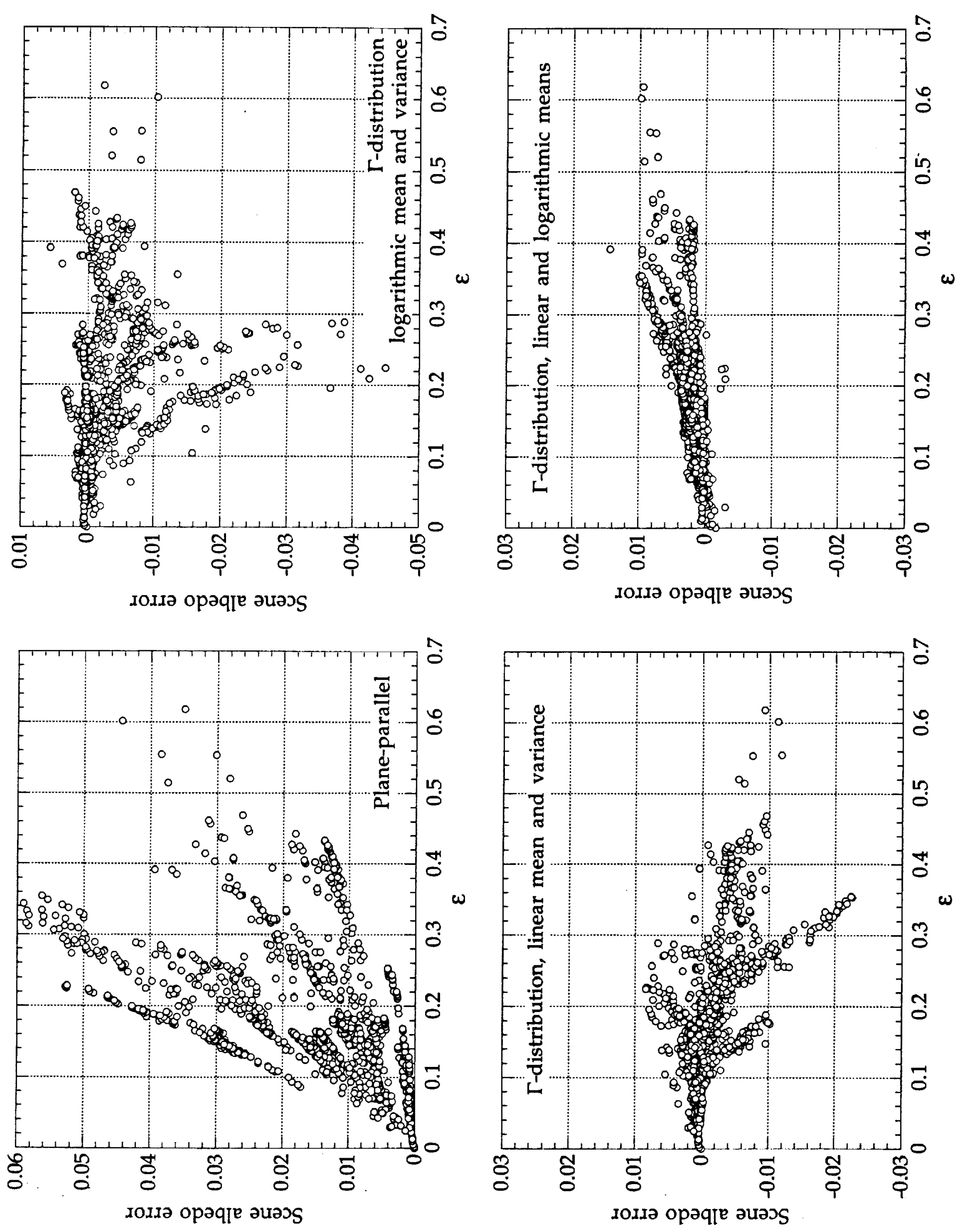